\title{
Low neoantigen expression and poor T cell priming underlie early immune escape in cancer
}

\section{Peter Westcott}

MIT https://orcid.org/0000-0001-9436-4857

\section{Nathan Sacks}

Massachusetts Institute of Technology

Jason Schenkel

MIT / Brigham and Women's Hospital

\section{Olivia Smith}

Massachusetts Institute of Technology https://orcid.org/0000-0002-2788-9917

\section{Daniel Zhang}

Massachusetts Institute of Technology

Haley Hauck

Massachusetts Institute of Technology

Coralie Backlund

Massachusetts Institute of Technology

\section{Mary Beytagh}

Massachusetts Institute of Technology

\section{JJ Patten}

Boston University https://orcid.org/0000-0002-9770-7525

\section{George Eng}

Koch Institute for Integrative Cancer Research at MIT

\section{Darrell Irvine}

Massachusetts Institute of Technology https://orcid.org/0000-0002-8637-1405

\section{Ömer Yilmaz}

Massachusetts Institute of Technology https://orcid.org/0000-0002-7577-4612

Tyler Jacks ( $\square$ tjacks@mit.edu )

Massachusetts Institute of Technology https://orcid.org/0000-0001-5785-8911

\section{Article}

Keywords: Immune Evasion, Immune Surveillance, Low Tumor Mutation Burden, Tumorigenesis 
DOI: https://doi.org/10.21203/rs.3.rs-70528/v1

License: (c) (1) This work is licensed under a Creative Commons Attribution 4.0 International License. Read Full License

Version of Record: A version of this preprint was published at Nature Cancer on September 30th, 2021. See the published version at https://doi.org/10.1038/s43018-021-00247-z. 
Low neoantigen expression and poor $\mathrm{T}$ cell priming underlie early immune escape in cancer

Peter M K Westcott ${ }^{1}$, Nathan Sacks ${ }^{1}$, Jason M Schenkel1,2, Olivia Smith¹, Daniel Zhang ${ }^{1}$, Haley Hauck ${ }^{1}$, Coralie

M. Backlund1, Mary C Beytagh1, JJ Patten¹, George Eng1,3, Darrell J. Irvine ${ }^{1,4,5,6,7}$, Omer H Yilmaz'1,3,8, Tyler $\operatorname{Jacks}^{1,7,8^{*}}$

\section{Affiliations}

${ }^{1}$ David H. Koch Institute for Integrative Cancer Research, Massachusetts Institute of Technology, Cambridge, MA 02139, USA

${ }^{2}$ Department of Pathology, Brigham and Women's Hospital, Boston, MA 02115, USA

${ }^{3}$ Department of Pathology, Massachusetts General Hospital, Boston, MA 02114, USA

${ }^{4}$ Department of Biological Engineering, Massachusetts Institute of Technology, Cambridge, MA 02139, USA

${ }^{5}$ Ragon Institute of Massachusetts General Hospital, Massachusetts Institute of Technology and Harvard University, Cambridge, MA 02139, USA

${ }^{6}$ Department of Materials Science and Engineering, Massachusetts Institute of Technology, Cambridge, MA 02139, USA

${ }^{7}$ Howard Hughes Medical Institute, Massachusetts Institute of Technology, Cambridge, MA 02139, USA

${ }^{8}$ Department of Biology, Massachusetts Institute of Technology, Cambridge, MA 02139, USA

*Corresponding author

\section{Abstract}

Immune evasion is a hallmark of cancer, and therapies that restore immune surveillance have proven highly effective in cancers with high tumor mutation burden (TMB) (e.g. microsatellite instable (MSI) colorectal cancer (CRC)). Whether low TMB cancers, which are largely refractory to immunotherapy, harbor $\mathrm{T}$ cell neoantigens capable of engaging adaptive immunity remains unclear. Here, we show that the majority of microsatellite stable (MSS) CRC harbors predicted high-affinity neoantigens despite low TMB. Unexpectedly, these neoantigens are broadly expressed at lower levels relative to those in MSI CRC, suggesting a potential role of antigen expression in tumor immune surveillance. To test this, we developed a versatile platform for functional interrogation of 
neoantigens with variable expression and applied it to novel preclinical colonoscopy-guided mouse models of CRC. While high expression of multiple high-affinity MHC-I-restricted neoantigens universally resulted in tumor rejection, low expression resulted in poor $\mathrm{T}$ cell priming and tumor progression. Strikingly, experimental or therapeutic rescue of priming rendered T cells fully capable of controlling tumors with low neoantigen expression. These findings underscore a critical role of neoantigen expression levels in immune evasion and suggest that poor expression or presentation may be a general feature of neoantigens acquired early in tumorigenesis. Finally, poorly expressed neoantigens, commonly excluded in tumor vaccine pipelines, may hold untapped therapeutic potential.

\section{Main}

Approximately $12 \%$ of $\mathrm{CRC}$ has defects in DNA mismatch repair (MMR) resulting in $\mathrm{MSI}^{1}$, with a high burden of mutation-derived tumor-specific antigens (neoantigens) that underlies favorable response to ICB ${ }^{2-4}$. The remaining majority of $\mathrm{CRC}$ is MSS with a lower TMB. On average, however, MSS CRC has more mutations than some cancers that respond favorably to $\mathrm{ICB}^{1,5}$. Indeed, our analysis of The Cancer Genome Atlas (TCGA) revealed that tumors from all MSS CRC patients expressed at least five neoantigens with strong predicted binding to their respective class I HLA ( $\left.\mathrm{IC}_{50} \leq 500 \mathrm{nM}\right)$, despite a lower TMB compared to MSI CRC patients (Fig. 1a). In addition, $68 \%$ of tumors from MSS patients expressed at least one neoantigen with predicted affinity as high or higher than the commonly-used model CD8 ${ }^{+} \mathrm{T}$ cell antigen SIINFEKL $\left(\mathrm{H}-2 \mathrm{~Kb} \mathrm{IC}_{50} \leq 10 \mathrm{nM}\right)^{6}$ (Fig. 1b). Unexpectedly, tumors from MSS patients had significantly lower median neoantigen expression compared to MSI patients (Fig. 1c). This difference is likely driven by the general enrichment of mutations in poorly-expressed genes in cancer ${ }^{7}$, a pattern absent in MMR-deficient tumors ${ }^{8}$. Together, this suggests the poor immunogenicity of MSS CRC is not due to complete absence of neoantigens but potentially their lower expression as well as colon-specific features that impinge on T cell immunity. Indeed, the intestinal microenvironment is tolerant to commensal bacteria and food-derived antigens, and these mechanisms may be co-opted by tumors to undermine immune responses ${ }^{9,10}$. In addition, the vast majority of $\mathrm{CRC}$ is associated with aberrant WNT/ $\beta$-catenin signaling ${ }^{1}$, which can promote exclusion of dendritic cells and failure to prime productive T cell responses in melanoma and liver cancer ${ }^{11,12}$. 


\section{Results}

Neoantigen expression level is a critical determinant of immune outcome in a novel orthotopic mouse model of colon cancer. To explore immune dysfunction in cancer, it is critical that preclinical models faithfully recapitulate the tissue microenvironment and genetics of the human disease. Likewise, to study processes underlying $\mathrm{T}$ cell dysfunction and immunotherapy resistance, models should enable manipulation of defined antigen-specific $\mathrm{T}$ cell responses. To our knowledge, no single model of $\mathrm{CRC}$ meets all of these criteria. Therefore, we adapted a technique employing endoscope-guided submucosal injection ${ }^{13}$ to induce geneticallydefined tumors in the mouse colon harboring model CD8 ${ }^{+} \mathrm{T}$ cell antigens. We first developed an autochthonous

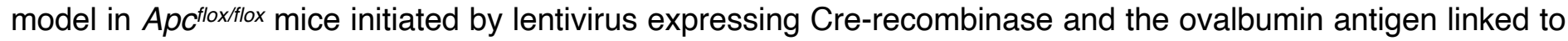
luciferase (LucOS) (Extended Data Fig. 1a), as our group has previously done in models of lung cancer ${ }^{14}$ and soft-tissue sarcoma ${ }^{15}$. Injection with LucOS dramatically reduced tumor incidence in a T cell-mediated manner, and tumors that did arise invariably lost antigen expression (Extended Data Fig. 1b-e). To assess effects of antigen expression in established tumors, T cells were continuously depleted for 5 weeks, at which point tumors retained antigen expression. However, 7 weeks after withdrawal of depleting antibodies, tumors had grown and lost antigen expression (Extended Data Fig. 1f-g). Given this potent immune editing and variability of antigen expression (Extended Data Fig. 1h), we developed an organoid model that maintains distinct levels of antigen expression throughout tumorigenesis. Organoid engineering also enables the use of a Kras mutant allele to model metastatic adenocarcinoma, which is confounded in the autochthonous model by concomitant Kras-driven fibrosarcoma formation (unpublished observations).

To enforce stable and continuous expression of antigen, we generated CRC organoids with SIINFEKL directly linked to Apc knockdown, an essential event in transformation. Specifically, we transformed normal

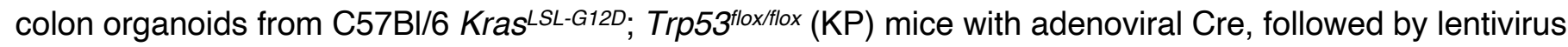
expressing miR-30 shRNA against $A p c(\operatorname{shApc})^{16}$ and SIINFEKL fused to the fluorophore mScarlet (mScarletsIIN) (Fig. 1d). Given that relief from Apc knockdown in shApc-transformed tumors results in regression ${ }^{16}$, dependence on shApc provides powerful selection against antigen loss (Extended Data Fig. 1i). Finally, deletion of Smad4, commonly mutated in CRC, was achieved by CRISPR-Cas9 editing. Selection of 
organoids harboring complete mutation of all genes was performed following published protocols ${ }^{17,18}$. This resulted in isogenic quadruple-mutant (shAKPS) organoids modeling the four most common genetic mutations in MSS CRC, which are co-mutated with high frequency in metastatic disease and associated with poor prognosis $^{19}$. To investigate the importance of neoantigen expression level, we generated organoids with 400fold range of mScarlet ${ }^{\text {SIIN }}$ expression via modifications to the shApc-expressing lentivirus, including placement in reverse orientation to the promoter (EF1a initiates bidirectional transcription), removal of the Kozak consensus sequence, and swapping $25 \%$ of codons with rare variants (Fig. 1d-e, Extended Data Fig. 1j). Importantly, this flexible system is broadly applicable to other cancers via linkage to relevant essential events (e.g. knockdown of Trp53) and is easily adapted to the study of other immune epitopes.

Endoscope-guided transplant of shAKPS organoids without antigen (no ${ }^{\mathrm{SIN}}$ ) efficiently induced tumors (Fig. 1f-g) and spontaneous metastasis to liver and lung, with histology remarkably similar to human CRC (Extended Data Fig. 1k). In contrast, transplant of the highest expression variant (hisıN) resulted in CD8+ T cellmediated rejection in all animals (Fig. 1f-g). We also generated shAKPS organoids harboring different epitopes with high affinity for MHC-I, including SIYRYYGL (hisIY), VGFNFRTL (hivGF), and ITYTWTRL (hilTY) (Extended Data Fig. 1I). The latter two are mutant epitopes of Lama4 (G1254V) and Alg8 (A506T) that arose in a methylcholanthrene-induced mouse sarcoma, which were reported to be insufficient for tumor rejection but central to ICB response in a syngeneic flank transplant model ${ }^{20}$. Here, all three epitopes resulted in tumor rejection (Extended Data Fig. 1m), demonstrating that immunogenicity is not idiosyncratic to SIINFEKL but a general feature of high expression of high-affinity epitopes. This also argues that the major genetic features of MSS CRC do not confer cell-autonomous resistance to T cell-mediated killing.

Strikingly, transplant of the lowest expression variant (loSIIN) induced tumors and metastases with similar efficiency, histology and infiltration as no $\mathrm{SIN}^{\mathrm{IN}}$ organoids (Fig. 1f-m). CD8 ${ }^{+} \mathrm{T}$ cells were sparse and only modestly increased in lo ${ }^{\mathrm{SIN}}$ tumors, while helper and regulatory $\mathrm{T}$ cell infiltration was not significantly different (Fig. 1j-n, Extended Data Fig. 1k). This is characteristic of the immune "cold" landscape of MSS CRC in humans ${ }^{21}$. Importantly, immune escape in lo ${ }^{\text {SIIN }}$ tumors did not result from neoantigen ignorance, as advanced tumors were infiltrated by antigen-experienced $\left(\mathrm{CD} 44^{+}\right)$and specific (SIINFEKL H-2Kb tetramer+) CD8 ${ }^{+} \mathrm{T}^{+}$cells (Fig. 10-p). Therefore, MSS tumors may harbor strong neoantigens despite poor T cell infiltration, consistent 
with our TCGA neoantigen analysis (Fig. 1a-b), and antigen expression level is a critical determinant of the anti-tumor immune response.

\section{Low neoantigen expression results in impaired T cell effector commitment and early dysfunction. To}

investigate why lo ${ }^{\mathrm{SIN}}$ tumors escaped immune rejection, we first compared the kinetics of the antigen-specific T cell response in lo ${ }^{\text {SIIN }}$ versus hiSIIN lesions. Low neoantigen expression resulted in both delayed and lower magnitude response (Fig. 2a). Interestingly, this difference was far less pronounced in the caudal and iliac draining lymph nodes (DLNs) (Extended Data Fig. 2a), suggesting that early lo ${ }^{\text {SIN }} \mathrm{T}$ cells are either impaired in their ability to traffic to or proliferate within the tumor. The latter is unlikely, however, as lo ${ }^{\text {SIIN }}$ and hisIN $T$ cells within tumors and DLNs showed no difference in proliferation (Extended Data Fig. 2b). Alternatively, T cells arriving at the tumor may have undergone deletional tolerance ${ }^{22}$, resulting in lower numbers. A critical step in the early maturation of functional T cell responses is effector differentiation, characterized by production of cytolytic granzymes and cytokines, and loss of progenitor potential23-25. We assessed levels of Granzyme B (GZMB) and TCF1, which is expressed in naïve, memory precursor and memory T cells and associated with stem-like properties ${ }^{23-25}$. Consistent with impaired effector differentiation, significantly more antigen-specific T cells from lo $\mathrm{SIIN}$ tumors and DLNs were TCF1+/GZMB', and significantly fewer were TCF1-/GZMB+, at 8 days (Fig. 2b,d-e, Extended Data Fig. 2c). This is unlikely a result of delayed kinetics, as the percentage of TCF1/GZMB + T cells in losIIN tumors at peak response (14 days) remained significantly lower (Fig. 2c,f, Extended Data Fig. 2d). Unexpectedly, the percentage of antigen-specific T cells capable of secreting both TNFa and IFNY effector cytokines was significantly higher in lo ${ }^{\text {SIIN }}$ versus hisIIN DLNs and not different in tumors at 8 days (Extended Data Fig. 2e). However, this cytokine-proficient population also showed higher TCF1 and lower GZMB (Extended Data Fig. 2f), suggesting similar lack of effector differentiation. Strikingly, the percentage of double-negative (TCF1-/GZMB') T cells was greater in lo ${ }^{\text {SIN }}$ versus hisIN animals at 8 days (Fig. 2b,g), and became even more pronounced by 14 days (Fig. 2c,h). Absence of TCF1 and GZMB indicates lack of progenitor or effector functionality and is associated with dysfunction. Indeed, by 14 days TCF1/GZMBantigen-specific T cells in lo ${ }^{\text {SIIN }}$ versus hisIIN tumors showed higher expression of co-inhibitory receptors PD-1, TIM3, LAG3, and 2B4 (Extended Data Fig. 2g), and an increased fraction co-expressing three or all four (Fig. 
2i, Extended Data Fig. 2h). To further interrogate functionality of the lo ${ }^{\text {SIIN }}$ response, we performed an in vivo killing assay ${ }^{26}$ by transferring SIINFEKL-loaded "target" splenocytes into tumor-bearing mice. Consistent with rejection of hisIN organoids, targets were nearly completely eliminated in the DLNs and spleen 6 hours posttransfer in hisIIN, but not lo ${ }^{\mathrm{SIIN}}$, animals at 8 days (Fig. 2j,k, Extended Data Fig. 2i). Even at the peak of the lo response (14 days), killing was incomplete (Fig. 2l,m, Extended Data Fig. 2j), with fewer targets killed per antigen-specific T cell (Fig. 2n). Altogether, these results demonstrate that low neoantigen expression drives an immediately dysfunctional T cell response with attenuated magnitude and per cell functionality.

T cells in tumors with low neoantigen expression become progressively dysfunctional. T cell dysfunction in human cancer is often attributed to upregulation of co-inhibitory receptors, terminal differentiation and loss of effector function, or "exhaustion"27,28. More recently, it has been shown that terminally-differentiated T cells in cancer are characterized by low TCF1 and high TIM3 expression, and are regenerated by "progenitor exhausted" T cells that have high TCF1 and low TIM3 expression ${ }^{29-31}$. Consistent with progressive dysfunction, terminallydifferentiated T cells (TCF1-/TIM3+) made up a greater proportion of the response at 42 compared to 8 days in Io SIIN tumors, but not in hisIN rejected lesions (Fig. 3a,b, Extended Data Fig. 3a). In contrast, progenitors (TCF1+/TIM3-) were substantially depleted in lo ${ }^{\text {SIIN }}$ tumors by 42 days (Fig. 3a,b, Extended Data Fig. 3b). In

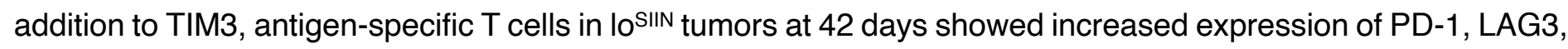
and TIGIT (Fig. 3c). In IoSIIN tumors, T cells negative for TCF1 and triple-positive for TIM3, PD-1, and LAG3 peaked at 42 days (Fig. 3d), consistent with progressive dysfunction. A similar trend was observed in

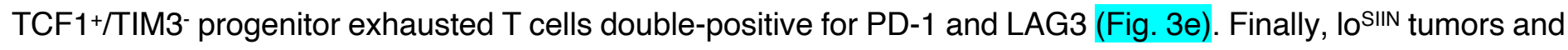

DLNs at 42 days showed a reduced percentage of TNFa and IFNY double-positive antigen-specific T cells (Extended Data Fig. 3c-d), indicating loss of effector functionality. Therefore, in addition to immediate dysfunction, T cells responding to low tumor neoantigen expression underwent progressive exhaustion.

\section{T cell priming is limiting in the context of low neoantigen expression and can be rescued by}

therapeutic vaccination. Impaired effector differentiation and early dysfunction are indicative of poor priming, such as occurs in the absence of CD4+ T cell "help"32. While Io ${ }^{\mathrm{SIIN}}$ and hisIN organoids lack a defined MHC-II- 
restricted model neoantigen, depletion of CD4+ $\mathrm{T}$ cells completely rescued formation of hisIIN tumors (Fig. 4a). Therefore, absence of help is unlikely the mechanism of dysfunction in the lo ${ }^{\text {IIIN }}$ model. Consistent with the importance of neoantigen expression level in priming, hisıN tumor formation was partially rescued in Batf3 ${ }^{-/}$ mice, which lack conventional cross-presenting dendritic cells (Fig. 4a). However, some Batf3 ${ }^{-/}$animals still rejected tumors, implicating additional antigen-presenting cells as important players in CD8+ ${ }^{+}$cell priming in the colon. To interrogate initial T cell activation, we performed a series of experiments with losIIN tumors in different priming contexts. First, we showed that in vitro-activated TCR-transgenic T cells specific to SIINFEKL (OT-1) were capable of killing lo $\mathrm{SIIN}_{\mathrm{N}}$ organoids when co-cultured (Fig. 4b-c). To optimize priming in vivo, we performed: 1) re-challenge with lo ${ }^{\text {SIIN }}$ organoids 28 days after "vaccination" with hisıIN organoids, 2) co-injection of lo ${ }^{\text {SIIN }}$ and hiSIIN organoids in the same animals, and 3) transfer of in vitro-activated OT-1 T cells concurrent with transplant of lo $\mathrm{SIIN}$ organoids. All of these approaches resulted in complete rejection of lo ${ }^{\text {SIIN }}$ organoids (Fig. 4d), demonstrating unequivocally that efficiently primed T cells are capable of killing tumor cells with low neoantigen expression in vivo. Priming in the context of high neoantigen also rescued the phenotype of T cells infiltrating lo ${ }^{\text {IIIN }}$ lesions. Notably, antigen-specific T cells infiltrating lo ${ }^{S I I N}$ and hisIN lesions from the same animals (Fig. 4e) showed similar abundance (Fig. 4f) and overlapping expression of TCF1 and GZMB that is indistinguishable from that of animals transplanted with only hisıN organoids (Fig. 4g, Extended Data Fig. 4a-c).

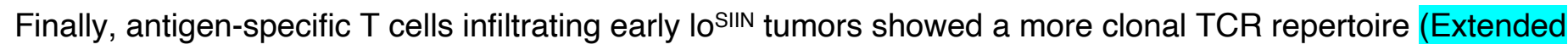
Data Fig. 4d-e), suggesting that poor priming limits the diversity of the T cell response.

Given the central role of T cell priming in tumor immune escape in our model, we tested the therapeutic potential of neoantigen vaccination in animals with established tumors. Mice with GFP-expressing tumors, as determined by colonoscopy, were randomly enrolled to receive SIINFEKL containing OVA $250-270$ (CGLEQLESIINFEKLTEWTSS) or non-specific mutant gp100 $20-39$ (CAVGALEGPRNQDWLGVPRQL) peptidebased vaccines consisting of a peptide-amphiphile and adjuvant amphiphile-CpG, which were administered at 14 and 21 days post-transplant. Vaccination with $\mathrm{OVA}_{250-270}$, but not non-specific peptide, induced profound expansion of tumor-specific T cells, with an average of $35 \%$ of peripheral blood CD8+ T cells displaying SIINFEKL specificity (Fig. 4h-I, Extended Data Fig. 4f-g). One week following the second dose, tumors in the OVA $_{250-270}$ vaccine arm showed significantly greater reduction in size relative to the non-specific arm, with 7 of 
$8(88 \%)$ showing a decrease in size and two undergoing complete regression (Fig. $4 \mathrm{j})$. These results argue that it is therapeutically tractable to vaccinate against poorly expressed neoantigens, and that strict neoantigen expression cutoffs in anti-tumor vaccine pipelines should be reevaluated.

\section{Agonistic CD40 rescues the poorly primed T cell response and enhances immune checkpoint blockade.}

We next asked whether more readily deployable antibody-based immunotherapies are efficacious in our model. Agonistic antibodies against the CD40 receptor (aCD40) enhance priming by potentiating the co-stimulatory function of antigen-presenting cells ${ }^{33}$. aCD40 is efficacious in preclinical mouse models of pancreatic ductal adenocarcinoma (PDAC), particularly when combined with ICB and immunogenic chemotherapy ${ }^{34}$. Recently, a phase lb clinical trial in PDAC with aCD40 (APX005M), aPD-1 (nivolumab), and gemcitabine/nab-paclitaxel has shown promising early results ${ }^{35}$. This is particularly exciting in light of the low TMB and immunogenicity of PDAC, which, like MSS CRC, is refractory to $\mathrm{ICB}^{36}$. Therapeutic combinations with aCD40 may be able to rescue or generate new $\mathrm{T}$ cell responses against weak affinity or poorly expressed neoantigens, or against tumorassociated self-antigens that lack high affinity $\mathrm{T}$ cell clones due to central tolerance. However, clinical studies in CRC are lacking.

We performed preclinical trials in mice bearing lo ${ }^{\text {SIIN }}$ colon tumors starting 14 days post-transplant (Extended Data Fig. 5a) with single agents aCD40, aPD-1, and aCTLA-4, as well as combinations aCD40/aPD1, aCD40/aCTLA-4, and aCD40/aPD-1/aCTLA-4. Response was evaluated by colonoscopy at 28 days posttransplant following Response Evaluation Criteria In Solid Tumors (RECIST) (Fig. 5g-h). All animals in the no treatment arm presented with progressive disease (Fig. 5a,i), while 2 of 12 (17\%), 3 of 12 (25\%), and 5 of 16 (31\%) showed complete responses in the aPD-1, aCTLA-4, and aCD40 arms, respectively (Fig. 5b-d). Response was notably better in all combination arms, with 9 of $12(75 \%), 8$ of $12(67 \%)$, and 12 of 17 (71\%) complete responses in the aCD40/aPD-1, aCD40/aCTLA-4, and aCD40/aPD-1/aCTLA-4 arms, respectively (Fig. 5e-f,i, Extended Data Fig. 5b). Comparing all combination arms as a whole against single agent aCD40 showed significantly more objective responses $(P=0.02)$ and complete responses $(P=0.01$, Fisher's exact test). Interestingly, adoptive cell transfer (ACT) of ex vivo-activated OT-1 T cells at 14 days post-transplant significantly 
delayed tumor growth but only resulted in one complete response (Extended Data Fig. 5c), suggesting that transferred T cells rapidly become dysfunctional.

Despite initially delayed tumor growth in the single ICB arms, no significant difference in final tumor burden was observed at necropsy, suggesting only transient effect in the majority of tumors (Fig. 5i,k). In addition, incidence of metastasis was not significantly decreased in single ICB arms (Fig. 5j,I-n). These results are reminiscent of the poor response to ICB seen in MSS CRC and demonstrate that ICB is only modestly effective at rescuing a poorly primed T cell response. In contrast, single agent aCD40 significantly decreased

primary tumor size at endpoint, while combination with ICB significantly reduced tumor size further (Fig. 5i). All treatment arms with aCD40 resulted in significantly reduced rates of metastasis (Fig. 5j), although this could reflect the absence of primary tumors in many of these animals. However, the combined rate of metastasis in animals with progressive disease across all aCD40 arms was still significantly reduced (Extended Data Fig. 5d). Interestingly, while ACT had no effect on reducing primary tumor size at endpoint, it resulted in complete control of metastatic tumor burden (Fig. 5j).

\section{Discussion}

The poor response of most CRC to immunotherapy represents a major unmet clinical need. Mouse models have provided invaluable insights into T cell dysfunction in cancer, but none to our knowledge recapitulate essential features of human CRC while facilitating detailed study of antigen-specific T cells. Here, we developed colonoscopy-guided models that enable comparison of functional versus dysfunctional tumorspecific T cell responses in a context highly faithful to the microenvironment, genetics, histopathology, and metastatic progression of the human disease.

We found that tumors from all MSS CRC patients in the TCGA harbored strong predicted neoantigens, but that these were broadly expressed at lower levels compared to those from MSI CRC. This raises the intriguing possibility that poor immunogenicity in MSS CRC and other immune cold cancers is driven by both lower burden and lower expression of neoantigens. Consistent with this notion, our low neoantigen-expressing model, like MSS CRC, demonstrated poor T cell infiltration and response to ICB. Leveraging the defined antigen in our model, we showed that low neoantigen expression precludes productive priming and drives a 
tolerogenic $\mathrm{T}$ cell response characterized by reduced magnitude, effector commitment, and per cell functionality. These findings provide broader context to previous flank transplant studies that found lower levels of epitope expression ${ }^{37}$ or $\mathrm{MHC}-\mathrm{I}$ binding affinity ${ }^{38}$ facilitate tumor immune escape, and a study that described early T cell dysfunction in an SV40 large T-antigen-driven model of liver cancer ${ }^{39}$. Indeed, a general feature of early immune evasion in many tumors may be that $\mathrm{T}$ cell dysfunction begins as a tolerogenic program initiated during priming with insufficient antigen stimulation, in addition to a lack of local inflammatory and/or costimulatory cues. By extension, it is likely that immune responses against clonal neoantigens in cancer, at least those acquired early in tumorigenesis, are poorly primed and tolerogenic-axiomatic to their failure to restrain tumor outgrowth. We showed that rescuing early priming is sufficient to prevent tumor initiation with $100 \%$ efficiency in our model, consistent with the fact that early neoplasia lack the immunosuppressive mechanisms to evade efficiently primed T cell responses. We also showed that poorly primed T cells in our model undergo progressive exhaustion, in line with prevailing literature and suggesting that $\mathrm{T}$ cell dysfunction in cancer is a heterogenous state shaped by multiple processes operative early and late in tumorigenesis. Future studies combining lineage tracing and single cell sequencing technologies should help disentangle the contribution of distinct genetic and epigenetic programs to T cell dysfunction in cancer.

Therapeutically targeting priming via aCD40 was highly efficacious in our model, particularly in combination with ICB, resulting in complete responses in the majority of animals. While ICB alone had no effect on the rate of metastasis, aCD40 and ACT almost completely prevented metastases, even in mice with progressive primary disease. Therefore, targeting priming may be especially efficacious against early metastatic lesions that may not be detected at the time of treatment. These results establish the preclinical utility of our model and highlight the therapeutic promise of combined aCD40 and ICB in the treatment of MSS $\mathrm{CRC}$ and other immune cold cancers. Furthermore, demonstration that anti-tumor immunity against a poorly expressed neoantigen can be rescued holds promise for therapeutic vaccination, justifying exploration of candidate neoantigens that by current practice are considered too poorly expressed. Finally, the flexible organoid-based system developed here should facilitate future studies of neoantigen expression level in faithful models of cancer, such as in modulating epitope immunodominance, and interplay of CD8+ and CD4+ neoantigen responses. 
Figure 1
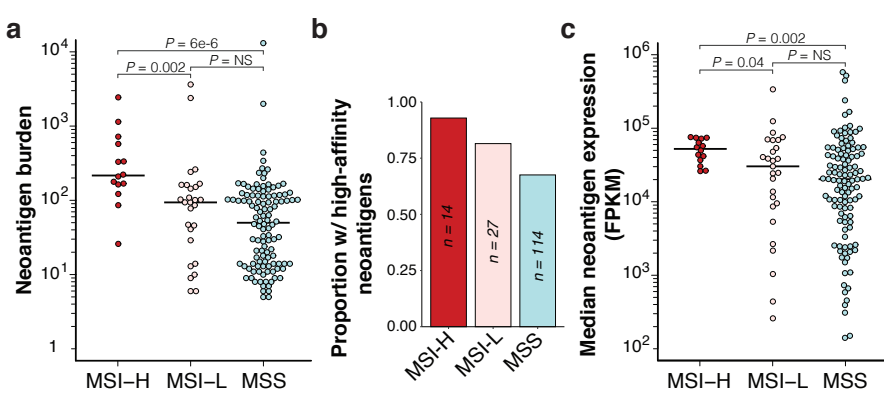

d no

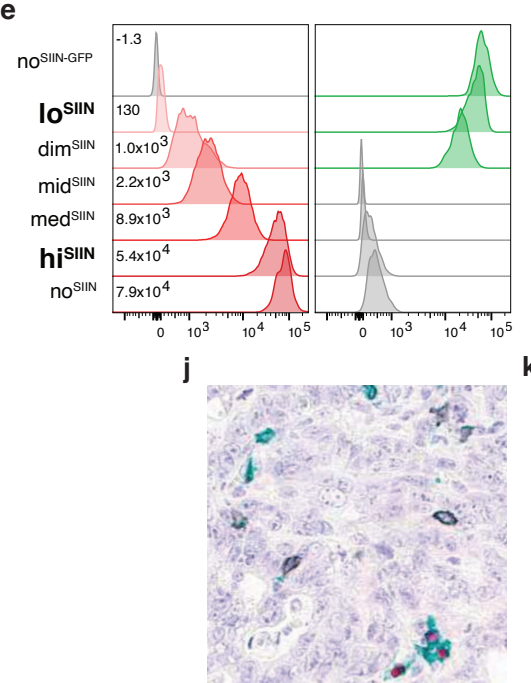

m
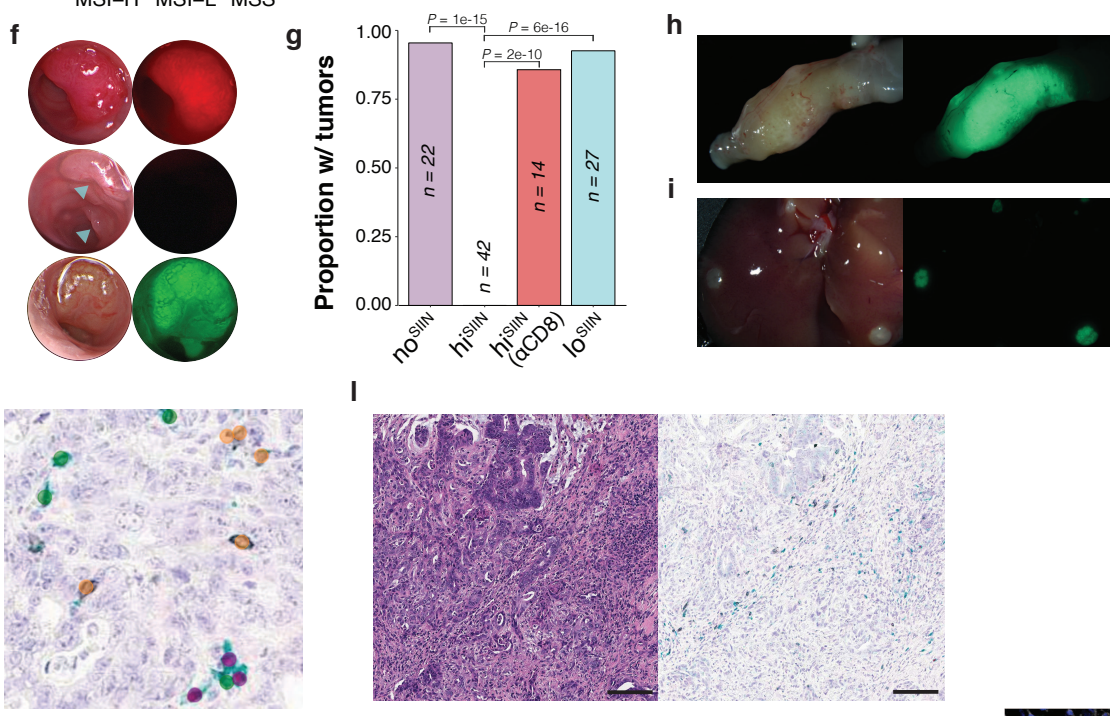

I
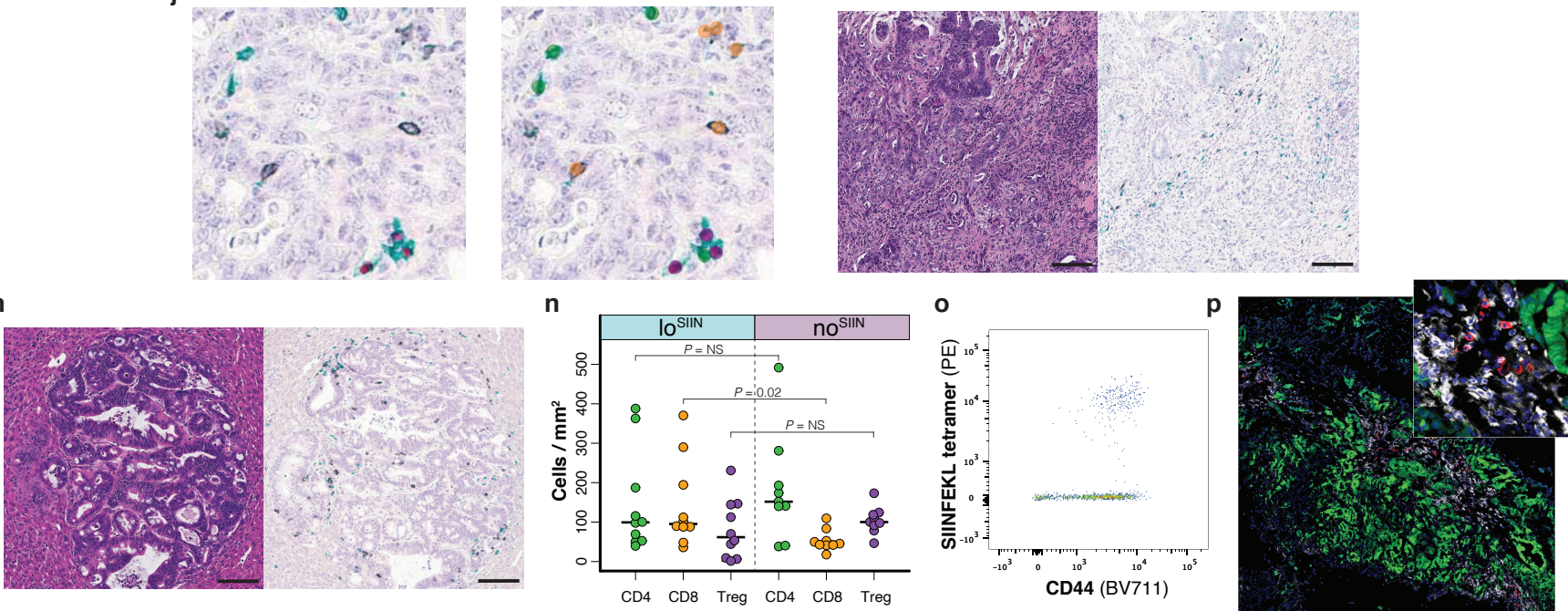
Figure $1 \mathrm{~A}$ novel model of $\mathrm{CRC}$ reveals a critical role of neoantigen expression level in tumor immune evasion. (a-c) Analysis of predicted neoantigens in human CRC (TCGA COADREAD) with high MSI (MSI-H), low MSI (MSI-L), and MSS. (a) Total expressed neoantigens with strong predicted HLA binding $\left(\mathrm{IC}_{50} \leq 500 \mathrm{nM}\right)$ by patient. (b) Proportion of patients expressing at least one neoantigen with predicted binding affinity equal to or greater than SIINFEKL ( $\left.\mathrm{IC}_{50} \leq 10 \mathrm{nM}\right)$. (c) Median expression of neoantigens by patient (FPKM, upper quartile-

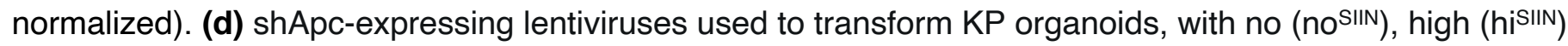
and low (IO ${ }^{\text {SIIN }}$ ) expression of SIINFEKL. Resulting shAKPS organoids were orthotopically-transplanted into the colons of syngeneic mice. (e) Expression of $\mathrm{mScarlet} / \mathrm{mScarlet}{ }^{\mathrm{SIIN}}$ and EGFP in expression variant organoids by flow cytometry. (f) Colonoscopy images of no ${ }^{\text {SIIN }}$ tumor (RFP channel), hisıIN scars (RFP channel, blue arrows indicate injection sites), and lo ${ }^{\text {IIIN }}$ tumor (GFP channel) 28 days post-transplant. (g) Efficiency of tumor formation 42 days post-transplant with no ${ }^{\mathrm{SIIN}}$, hiSIIN, hiSIIN with antibody depletion of CD8+ T cells, and Io ${ }^{\text {SIIN }}$ organoids. (h-i) Stereoscopic brightfield and fluorescent images of losIIN colon tumor (h) and liver metastases (i) 42 days post-transplant. (j-k) Example three color IHC (black = CD8, green = CD4, red = FOXP3) (J) and automated annotation by convolutional neural network (k). (I-m) Representative H\&E and three color IHC of IOSIIN primary colon tumor (I) and liver metastasis (m) 42 days post-transplant. Scale bar = $100 \mu \mathrm{M}$. (n) Quantification of CD8, CD4, and Treg cells infiltrating lo ${ }^{\text {IIN }}$ and no ${ }^{\text {SIIN }}$ tumors by convoluted neural network analysis. Each point represents at least one tumor from a single animal. (o-p) Identification of SIINFEKL tetramer-specific CD8+ T cells infiltrating 42 day losIIN tumors by flow cytometry (o) and immunofluorescence (p) with in situ tetramer staining (green $=$ tumor, white $=$ CD8, red $=$ SIINFEKL tetramer, blue = DAPI). 


\section{Figure 2}

a

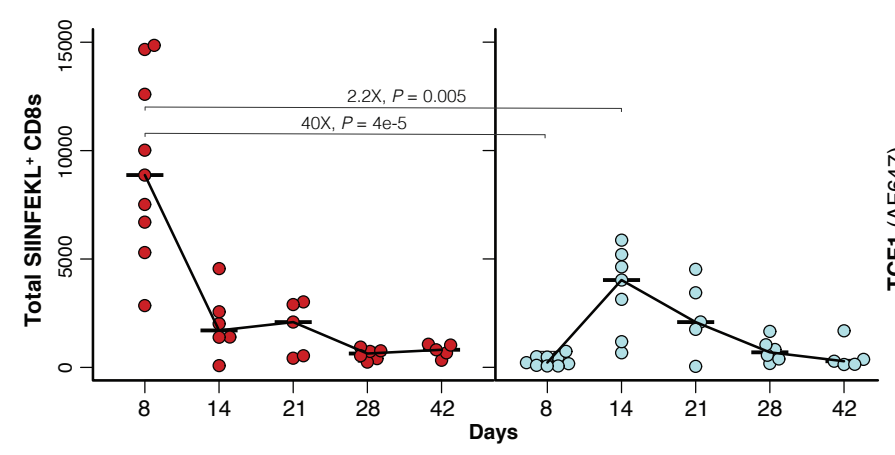

e

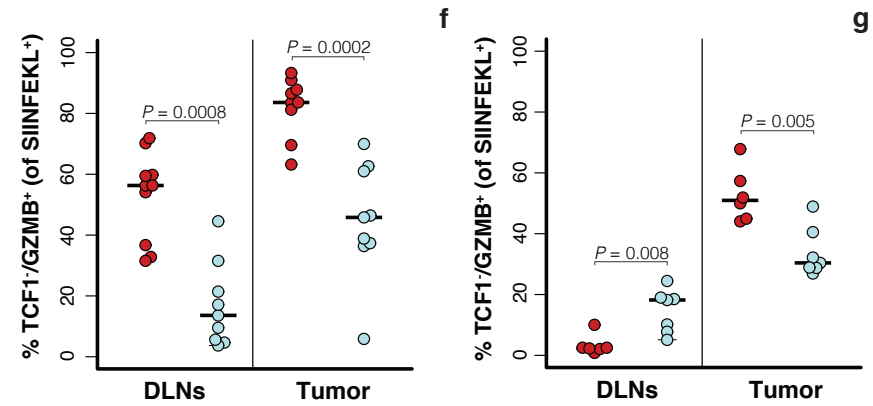

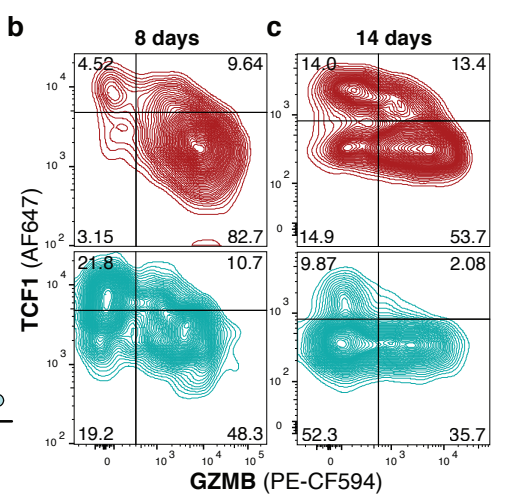

d

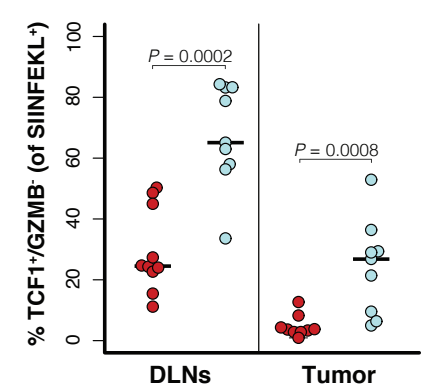

h

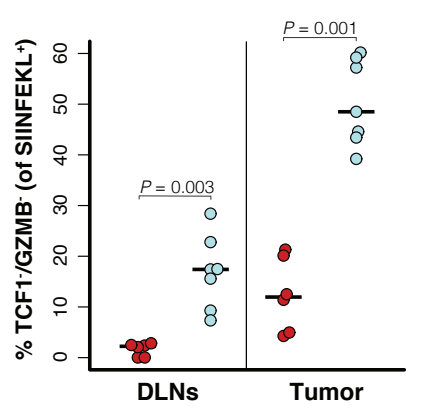

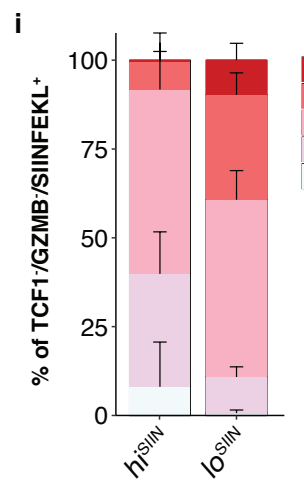
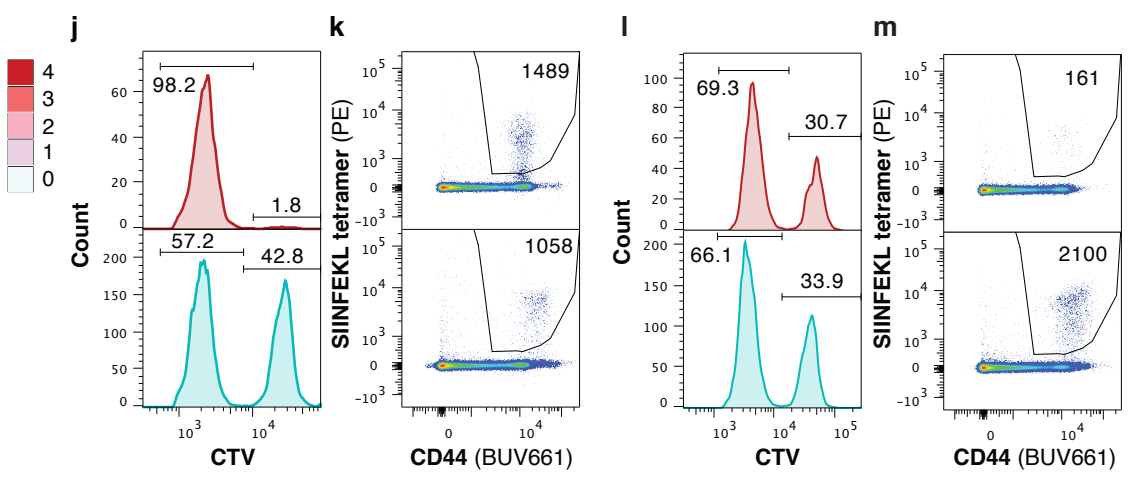

n

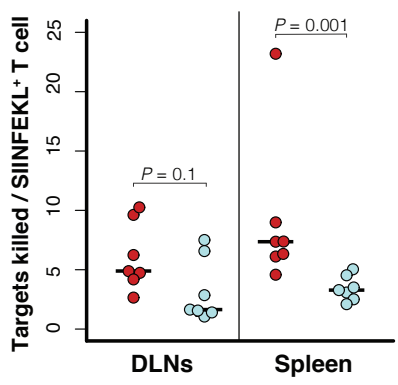


Figure 2 Low neoantigen expression drives impaired T cell effector commitment and immediate dysfunction.

(a) Total numbers of $\mathrm{CD} 44^{+} / \mathrm{CD} 8^{+}$antigen-specific $\mathrm{T}$ cells isolated from tumors at $8,14,21,28$, and 42 days post-transplant of hisIIN (red) and loSIIN (blue) organoids by flow cytometry. (b-c) Antigen-specific T cell expression of TCF1 versus GZMB in tumors at 8 (b) and 14 (c) days. (d-e,g) Percent of antigen-specific T cells from DLNs and tumors at 8 days positive for TCF1 and negative for GZMB (d), negative for TCF1 and positive for GZMB (e), and double-negative for TCF1 and GZMB (g). (f,h) Percent of antigen-specific T cells from DLNs and tumors at 14 days negative for TCF1 and positive for GZMB (f), and double-negative for TCF1 and GZMB (h). (i) Percentage of TCF1/GZMB- antigen-specific T cells from tumors at 14 days expressing $0,1,2,3$, and 4 inhibitory receptors (PD-1, TIM3, LAG3, and 2B4). (j-m) Representative in vivo killing assay histograms of transferred control (weak CTV stain) and SIINFEKL-loaded "target" (strong CTV stain) splenocytes and flow plots of antigen-specific T cells recovered from DLNs at $8(\mathbf{j}-\mathbf{k})$ and 14 (I-m) days post-transplant of hisinN (red) and lo ${ }^{\text {SIIN }}$ (blue) organoids. Percent of control and target populations are noted in histograms, and total number of antigen-specific T cells noted in flow plots. (n) Target killing normalized to number of antigen-specific $\mathbf{T}$ cells recovered in 14 day in vivo killing assay. 
Figure 3
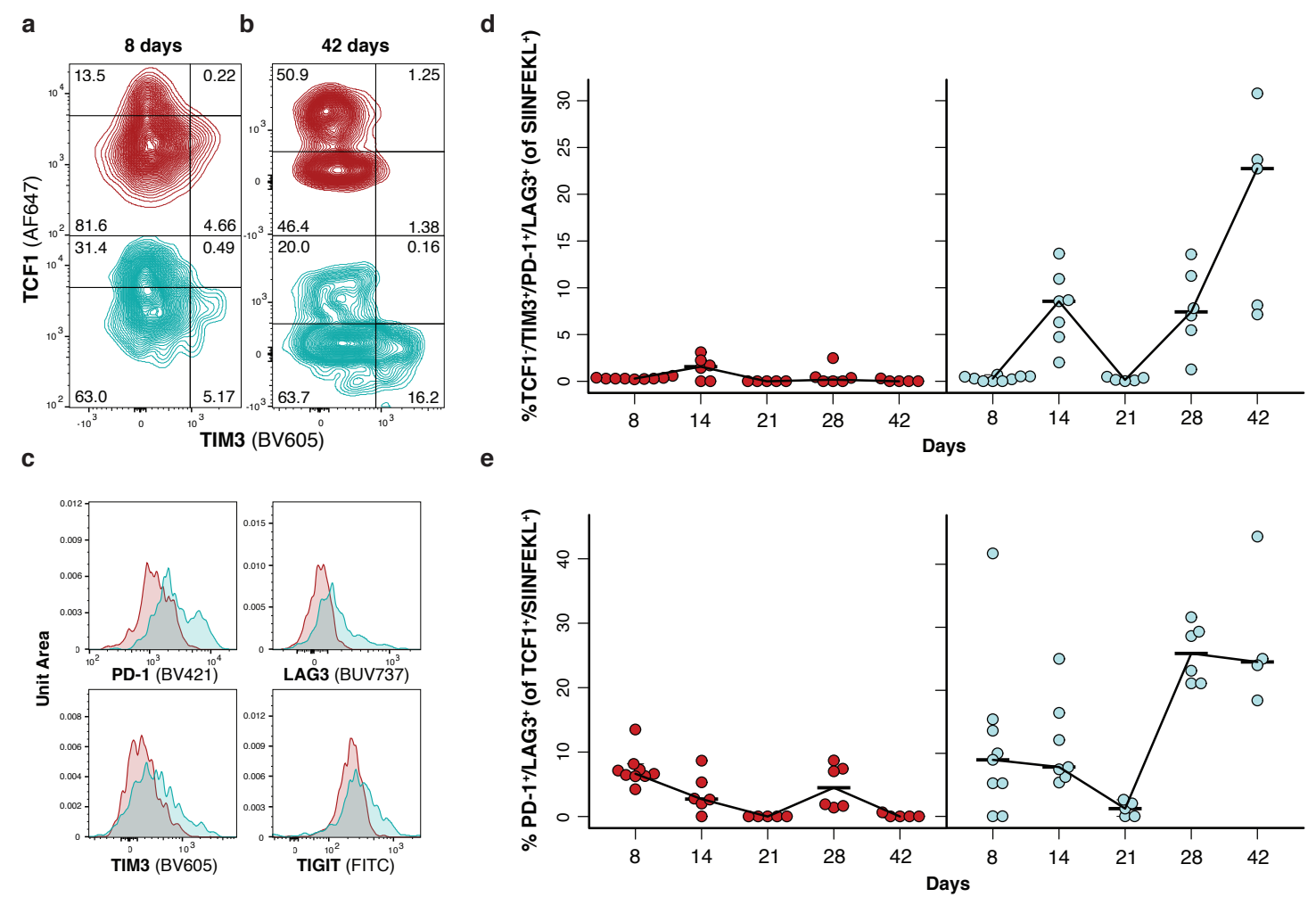
Figure 3 T cells in tumors with low neoantigen expression become progressively dysfunctional.

(a-b) Antigen-specific T cell expression of TCF1 versus TIM3 in tumors at 8 (a) and 42 (b) days posttransplant. (c) Representative histograms of inhibitory receptor expression on antigen-specific $T$ cells from tumors at 42 days post-transplant. (d-e) Percent TCF1-/TIM3+/PD-1+/LAG3+ (d) and TCF1+/PD-1+/LAG3+ (e) antigen-specific $T$ cells isolated from tumors at $8,14,21,28$, and 42 days post-transplant. Red $=$ hisıN, blue $=$ IOSIIN. 


\section{Figure 4}

a
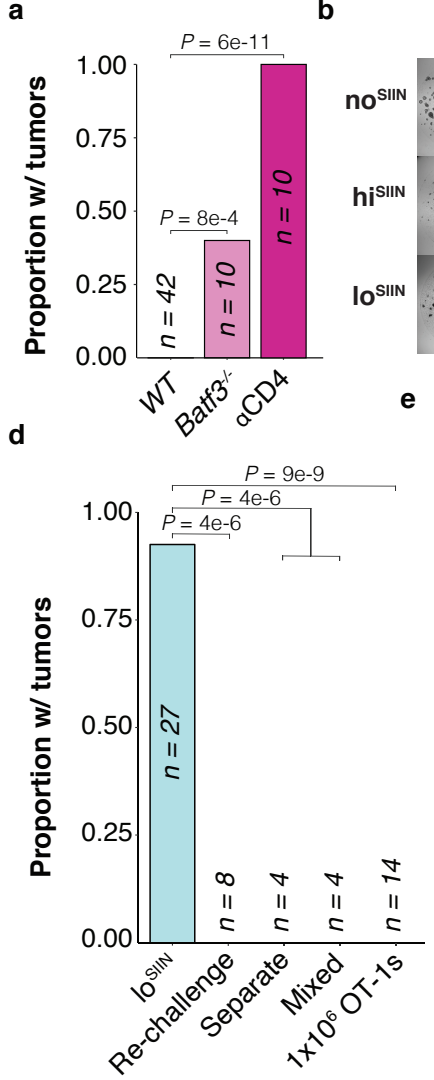

h
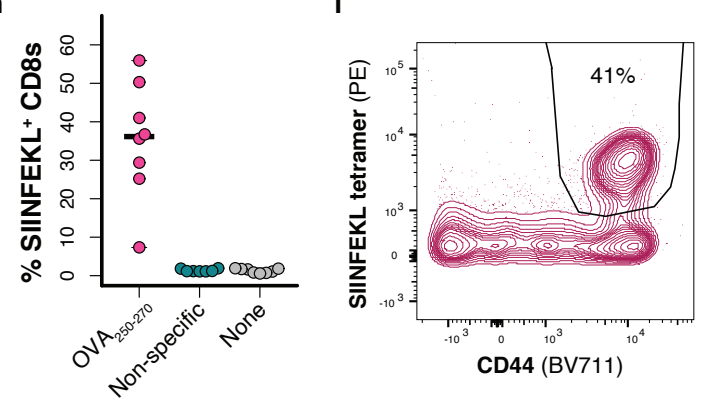

c
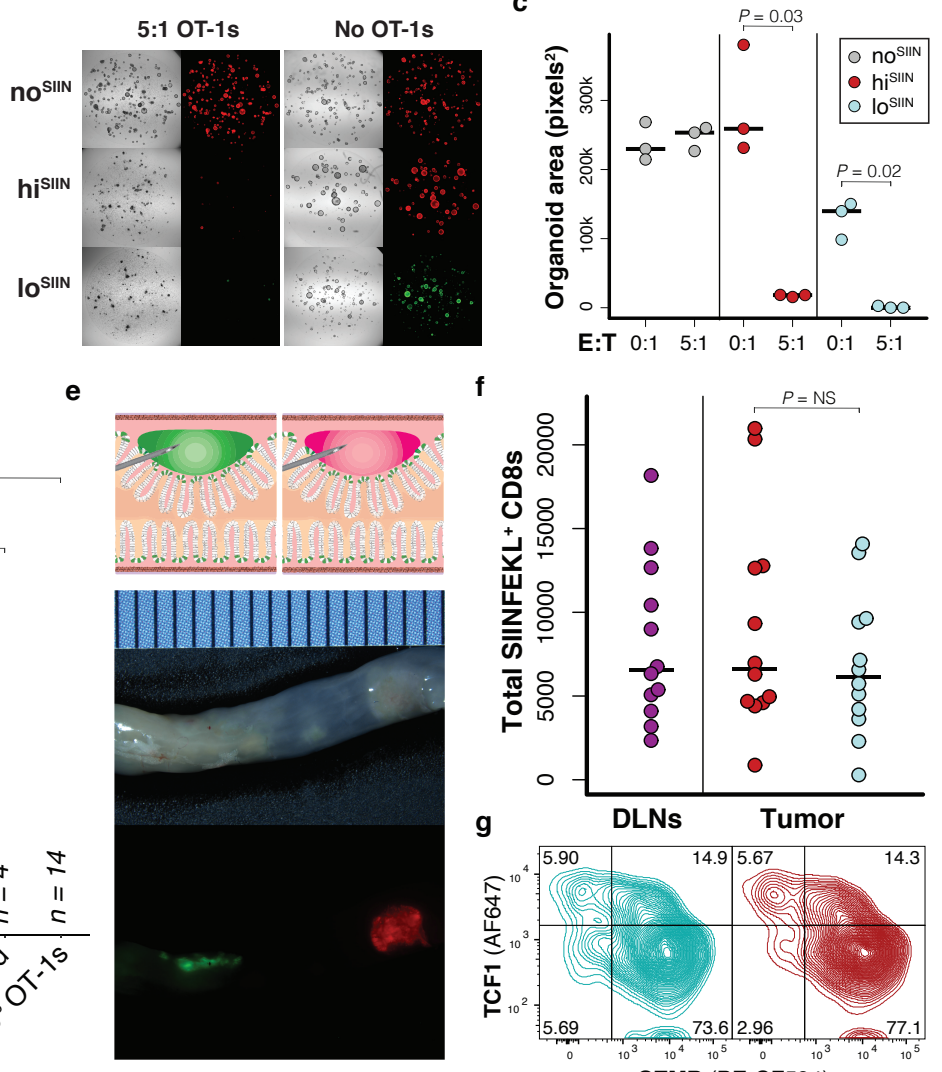

GZMB (PE-CF594)

j

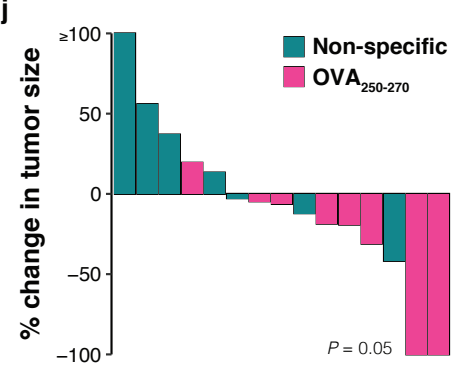


Figure 4 Low neoantigen expression results in poor T cell priming that can be rescued by therapeutic vaccination. (a) Efficiency of tumor formation 6 weeks post-transplant of hisIIN organoids into wild-type (WT) and Batf3 knockout mice, and 3 weeks post-transplant into WT mice with continuous antibody depletion of CD4+ T cells. (b) Representative images of co-cultures with no ${ }^{\mathrm{SIIN}}$, hiSIIN, and Io ${ }^{\mathrm{SIIN}}$ organoids and in vitroactivated OT-1s at day 4. (c) Quantification of co-culture organoid confluence. E:T = effector to target ratio. (d) Efficiency of tumor formation 6 weeks post-transplant of loSIIN, loSIIN 4 weeks after transplant of hisIN $(R e-$ challenge), lo ${ }^{\text {SIIN }}$ and hiSIIN at separate sites in the same animal (Separate), lo ${ }^{\text {SIIN }}$ and hisIN at the same sites (Mixed), and lo ${ }^{\text {SIIN }}$ concurrent with retro-orbital injection of $1 \times 10^{6}$ in vitro-activated OT-1s. (e) Schematic of transplant of lo ${ }^{\mathrm{SIIN}}$ (green) and hisIN (red) organoids at separate sites in the distal colon of the same animal (top), and stereoscopic brightfield (middle) and fluorescent (bottom) images of a co-transplanted colon 8 days post-transplant. (f-g) Flow cytometric analysis of antigen-specific T cells isolated from DLNs and tumors 8 days post-co-transplant of hisIN (red) and lo ${ }^{\text {SIIN }}$ (blue) organoids at separate sites in the same animals. (f) Total number of antigen-specific T cells. (g) Representative expression of GZMB versus TCF1. (h) Percent of total peripheral blood CD8 ${ }^{+}$T cells that are antigen specific (CD44+/SIINFEKL tetramer ${ }^{+}$) following two weeks (two doses) of $\mathrm{OVA}_{250-270}$, non-specific, or no peptide-based vaccination in losIIN tumor-bearing mice. (i) Representative flow plot of peripheral blood antigen-specific CD8 ${ }^{+} T$ cells from $\mathrm{OVA}_{250-270}$ vaccinated mouse.

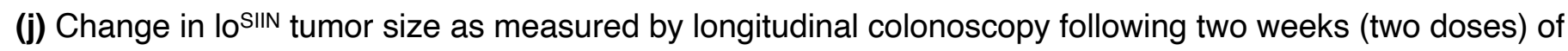
$\mathrm{OVA}_{250-270}$ or non-specific vaccination. Significance was assessed by Wilcoxon rank-sum test of percent change in tumor size. 
Figure 5
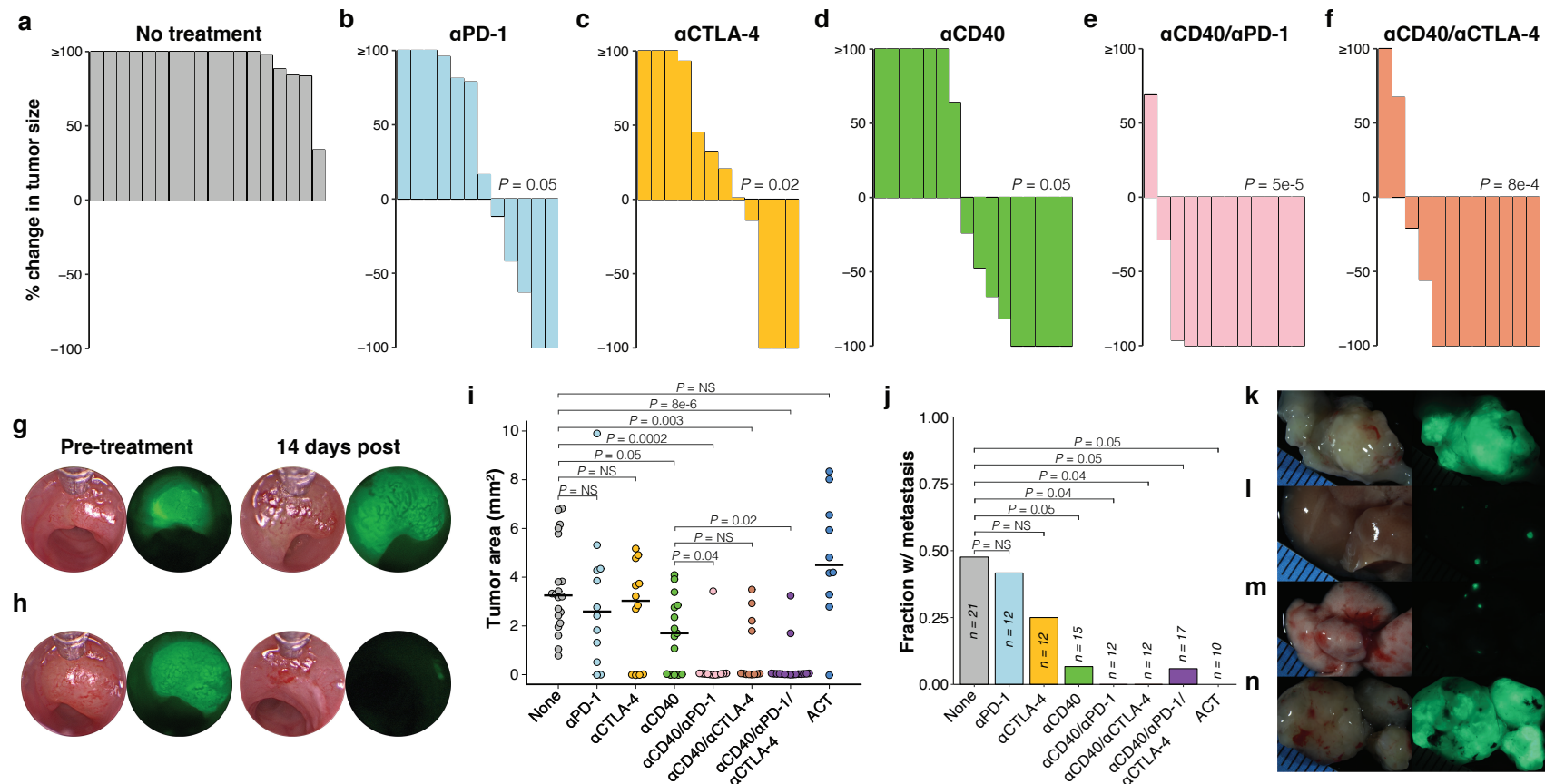
Figure 5 Agonistic CD40 rescues deficient priming and enhances checkpoint blockade in low neoantigenexpressing tumors. (a-f) Preclinical trial of mice bearing lo ${ }^{\text {SIIN }}$ tumors. Mice were recruited into treatment arms at 14 days post-transplant. Waterfall plots show change in tumor size after 14 days of treatment, as determined by colonoscopy. (g-h) Representative colonoscopy white light and fluorescent images of tumors pre- and posttreatment from mice receiving no treatment (g) and aCD40/aPD-1/aCTLA-4 (h). (i) Primary tumor sizes at necropsy 28 days post-treatment initiation. ACT = adoptive cell transfer of OT-1s. (j) Fraction of mice with any metastases (liver, lung, or omentum). (k-n) Representative stereoscopic white light and fluorescent images of primary colon tumor (k), liver ( $\mathbf{l})$, lung $(\mathbf{m})$, and omental $(\mathbf{n})$ metastases from an aPD-1-treated mouse 28 days post-treatment initiation. 
Extended Data Figure 1

a
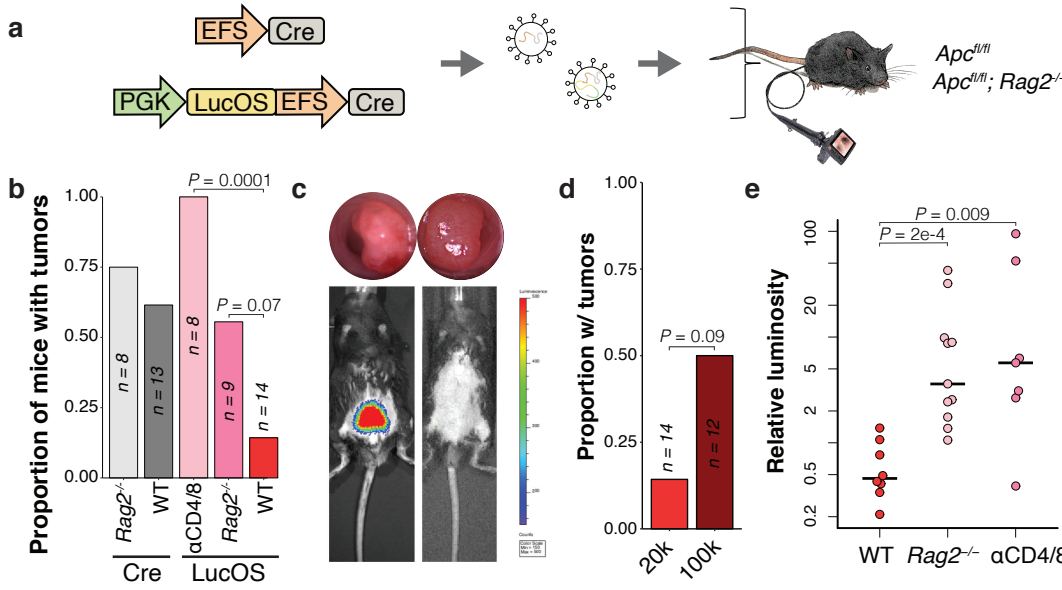

e

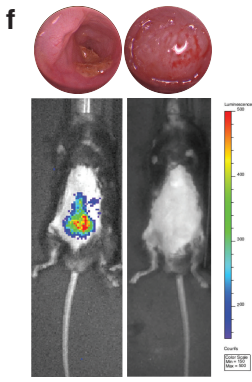

g

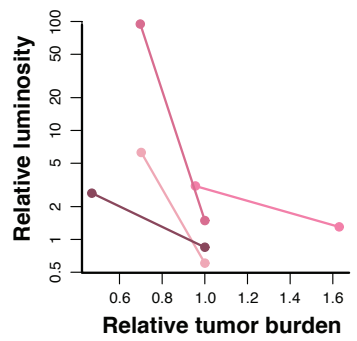

h
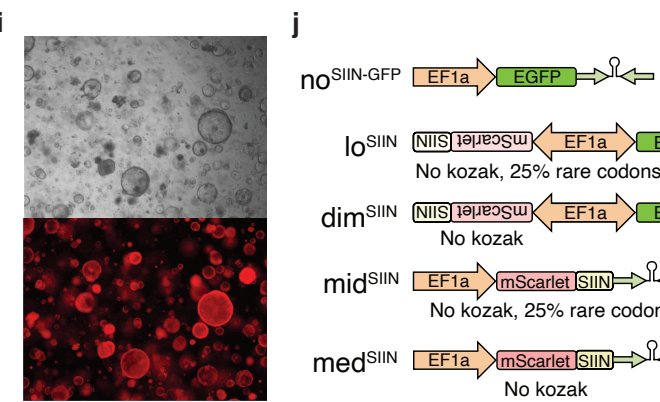

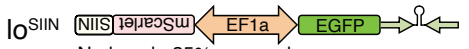
No kozak, $25 \%$ rare codons

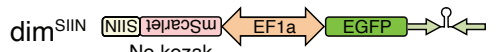

mid $^{\text {SIIN }} \underset{\text { EF1a }}{ }>$ mScarlet SIIN $\Rightarrow R_{\hookleftarrow}$

No kozak, $25 \%$ rare codons

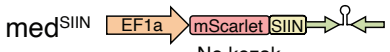

I No kozak

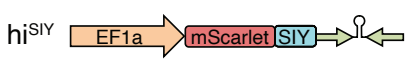

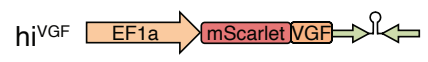

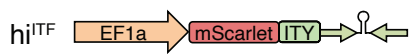

m

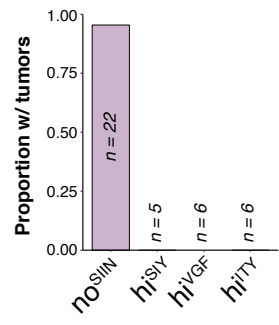

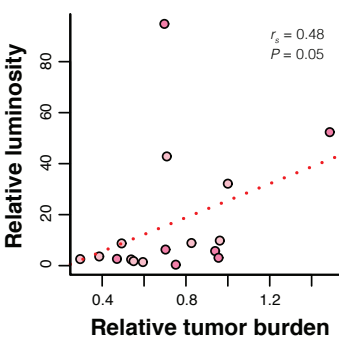

k
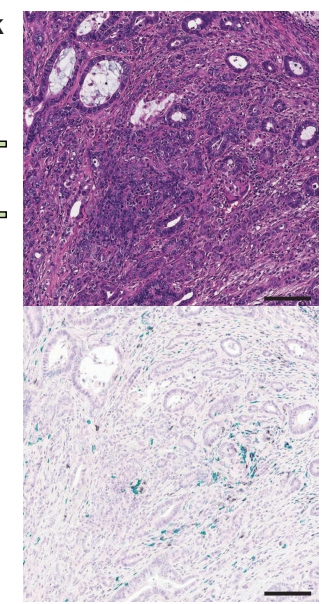

$$
-
$$


Extended Data Figure $1 \mathrm{~A}$ novel model of $\mathrm{CRC}$ reveals a critical role of neoantigen expression level in tumor immune evasion. (a) Cre and LucOS lentiviruses used to initiate colon tumors in Apc floxfllox and Apc floxfllox; Rag2/- mice. (b) Efficiency of tumor formation 16 weeks post-injection. (c) Antigen expression is maintained in LucOS-induced tumors in Rag2\% mice (left), but not in rare LucOS tumors in wild-type mice (right) at 12 weeks (colonoscopy above, in vivo bioluminescence below). (d) Injection of LucOS lentivirus at higher titer $(100,000$ versus $20,000 \mathrm{TU} / \mu \mathrm{l}$ ) results in greater efficiency of tumor induction. (e) Antigen expression is lost in all LucOS tumors formed in wild-type mice, regardless of titer, but maintained in Rag2 $2^{-/}$mice and mice receiving continuous antibody depletion of $\mathrm{CD}^{+}$and $\mathrm{CD} 8^{+} \mathrm{T}$ cells. (f) Antigen expression is maintained in LucOSinduced tumors with continuous T cell depletion at 5 weeks (left), but lost 7 weeks after withdrawal of T cell depleting antibodies, despite continued tumor growth (right). (g) Loss of antigen following withdrawal of T cell depleting antibodies, despite increased tumor size (as measured by percent of colonic lumen occluded by tumor). (h) Relatively poor correlation of antigen expression and tumor burden (by colonoscopy) in Rag2 $2^{-1-}$ (dark pink) or aCD4/8 (light pink)-treated mice induced with LucOS at 12 weeks post-injection. (i) KP organoids transformed with mScarlet-shApc lentivirus grow in the absence of Wnt and are uniformly red fluorescent. (j) Lentiviral constructs used to generate control organoids expressing only EGFP (noSIIN-GFP), and SIINFEKL expression variants employing forward and reverse orientation to promoter, no kozak, $25 \%$ rare codons, or all three. (k) Representative H\&E and three color IHC (black = CD8, green = CD4, red = Foxp3) of no ${ }^{\text {SIIN }}$ primary colon tumor 42 days post-transplant. Scale bar $=100 \mu \mathrm{M}$. (I) Lentiviral constructs used to generate organoids expressing the epitopes SIYRYYGL, VGFNFRTL, and ITYTWTRL. (m) Efficiency of tumor formation 42 days

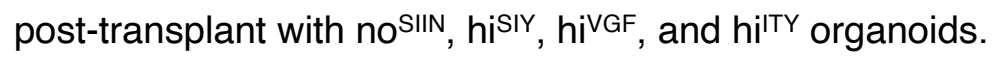




\section{Extended Data Figure 2}
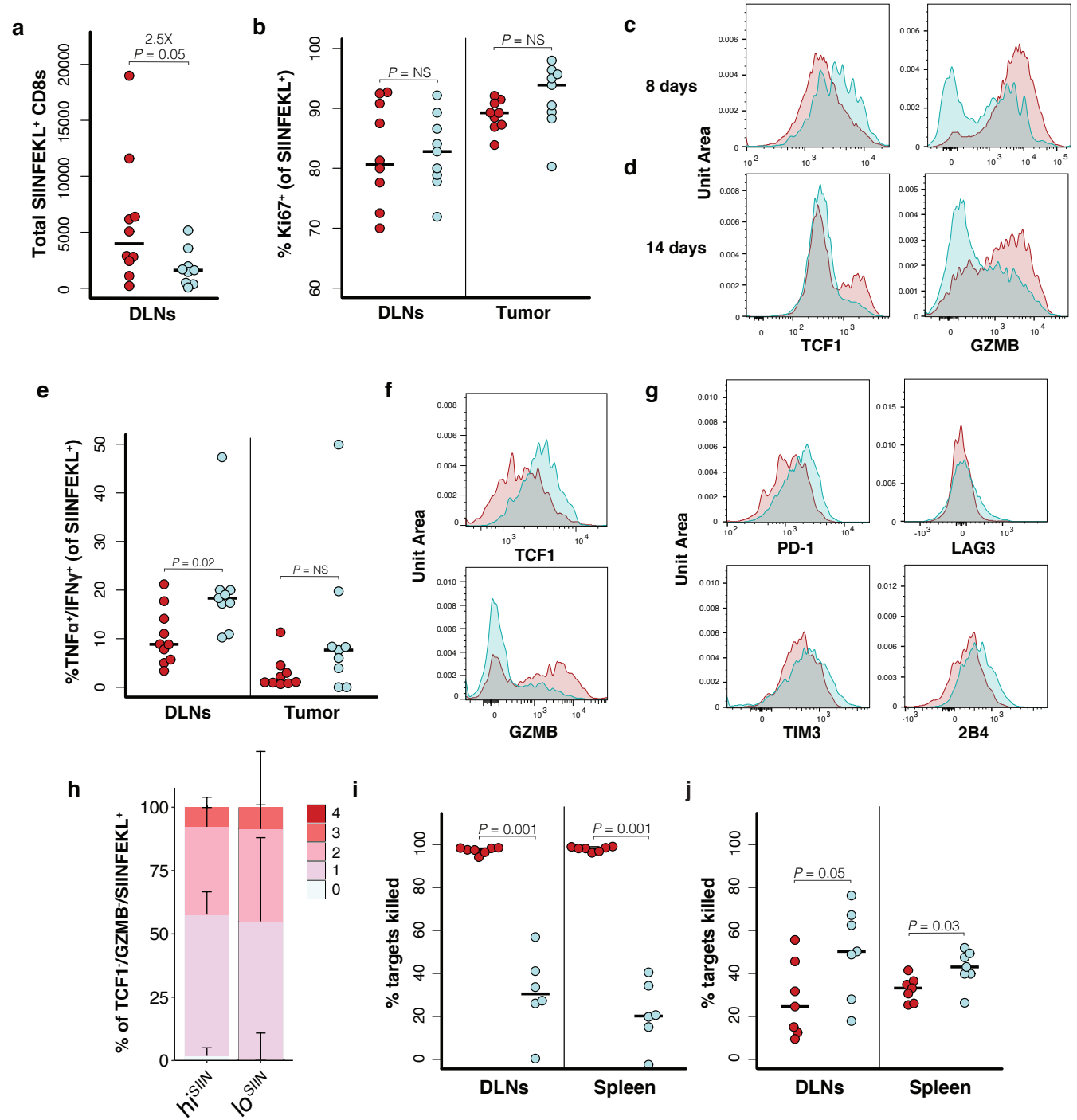
Extended Data Figure 2 Low neoantigen expression drives impaired T cell effector commitment and immediate dysfunction. (a-m) Flow cytometry of CD44+/CD8 ${ }^{+}$antigen-specific T cells from tumors and DLNs post-transplant of hisIIN (red) and lo ${ }^{\text {SIN }}$ (blue) organoids. (a) Total number of antigen-specific T cells in DLNs at 8 days, and (b) the percent that are positive for Ki67. (c-d) Representative histograms of TCF1 and GZMB expression in antigen-specific T cells in tumors at 8 (c) and 14 (d) days. (e) Percent of antigen-specific T cells at 8 days double-positive for TNFa and IFNY. (f) Representative histograms of TCF1 and GZMB expression on TNFa/IFNy double-positive antigen-specific T cells within DLNs. (g) Representative histograms of inhibitory receptor expression on TCF1/GZMB- antigen-specific T cells from tumors at 14 days. (h) Percent of TCF1/GZMB- antigen-specific T cells from tumors expressing 0, 1, 2, 3, and 4 inhibitory receptors (PD-1, TIM3, LAG3, and 2B4) at 8 days. (i-j) Percent of SIINFEKL-loaded "target" splenocytes killed in DLNs and spleens from in vivo killing assay at 8 (i) and 14 (j) days post-transplant of hiSIN and lo ${ }^{\text {IIN }}$ organoids. 

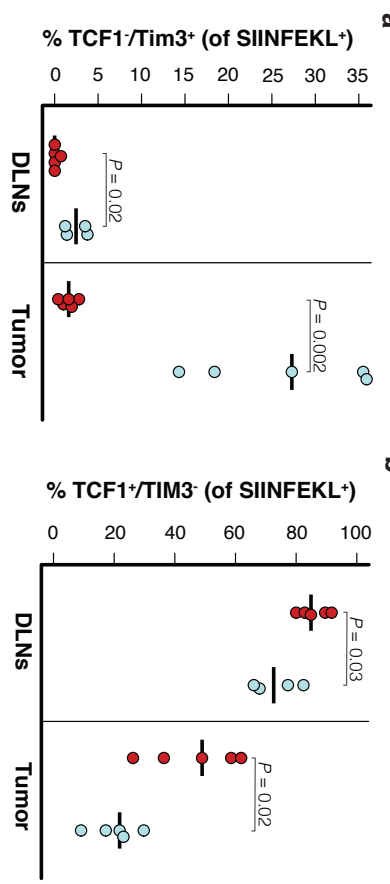

$\% \mathrm{TNFa}^{+} / \mathrm{IFNY} \mathbf{Y}^{+}$(of SIINFEKL+)

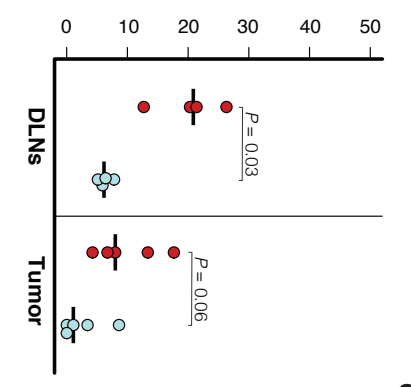

$\% \mathrm{TNFa}^{+} / \mathrm{FNY}^{+}$(of SIINFEKL+)

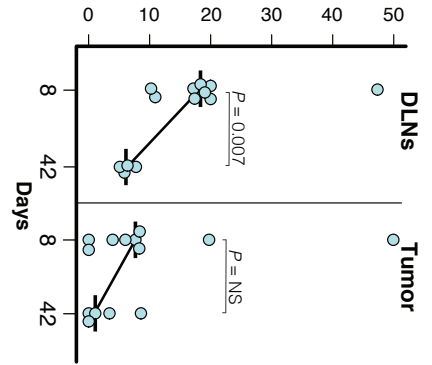


Extended Data Figure 3 T cells in tumors with low neoantigen expression become progressively

dysfunctional. (a-b) Percent of antigen-specific T cells from DLNs and tumors at 42 days negative for TCF1 and positive for TIM3 (a), and positive for TCF1 and negative for TIM3 (b). (c-d) Percent of antigen-specific T cells from DLNs and tumors double-positive for TNFa and IFNY at 42 days (c) and both 8 and 42 days (d). Red $=$ hisIIN, blue $=10^{\text {SIIN }}$. 


\section{Extended Data Figure 4}

a
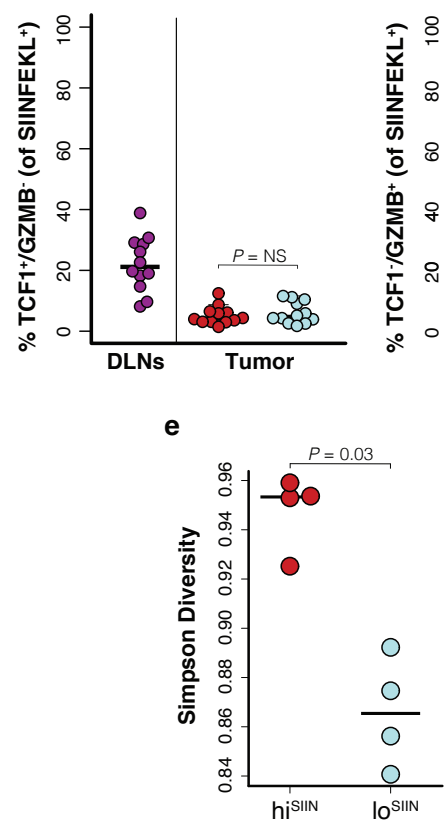

b

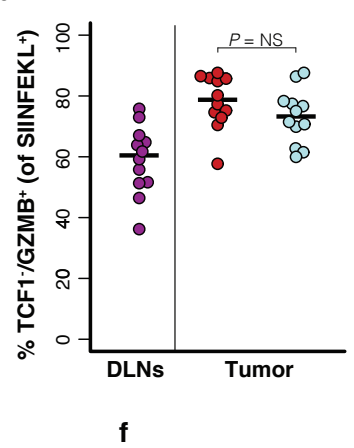

c

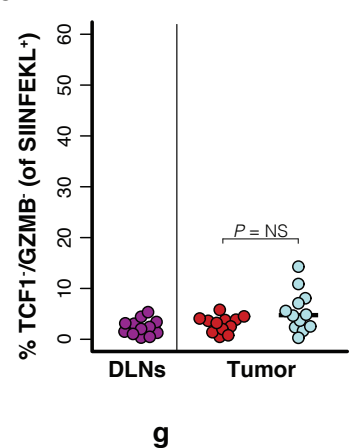

d

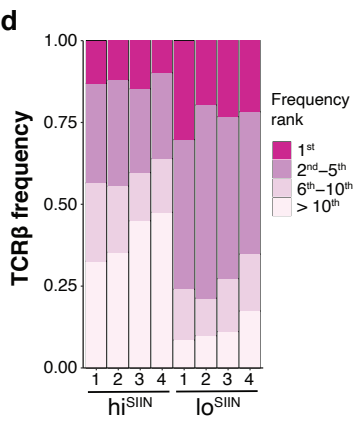

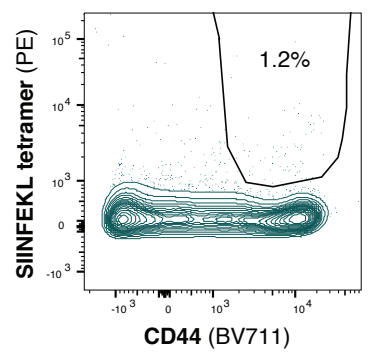

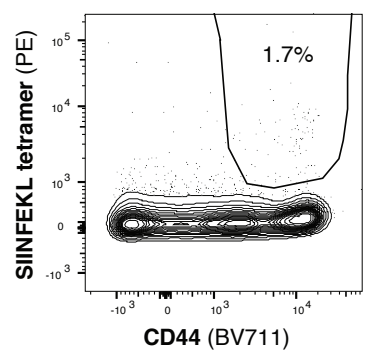


Extended Data Figure 4 Low neoantigen expression results in poor T cell priming that can be rescued by therapeutic vaccination. (a-c) Flow cytometric analysis of antigen-specific T cells from DLNs and tumors 8 days post-co-transplant of hisIN (red) and lo ${ }^{\text {SIIN }}$ (blue) organoids at separate sites in the same animals. Percent positive for TCF1 and negative for GZMB (a), negative for TCF1 and positive for GZMB (b), and doublenegative for TCF1 and GZMB (c). Frequency of the most common clonotypes (d) and Simpson diversity score (e) across four biological replicates of TCR $\beta$ chain sequencing of antigen-specific T cells from hiSIIN and Io ${ }^{\text {IIIN }}$ tumors at 8 days. (f-g) Representative flow plots of peripheral blood antigen-specific (CD44+/SIINFEKL tetramer $^{+}$) CD8+ $\mathrm{T}$ cells from non-specific peptide-based vaccination (f) and no vaccination control (g) mice. 
Extended Data Figure 5
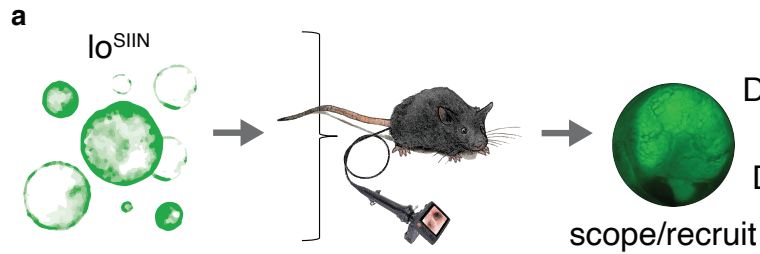
$\begin{array}{llllllllllll}\text { Dose: } 1 & 2 & 3 & 4 & 5 & 6 & 7 & 8 & 9 & 10 & 11 & 12\end{array}$ $\begin{array}{lllllllllllll}\text { Day: } 14 & 16 & 18 & 21 & 23 & 25 & 28 & 30 & 32 & 35 & 37 & 39 & 42\end{array}$

b

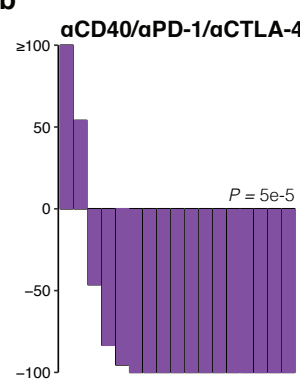

c

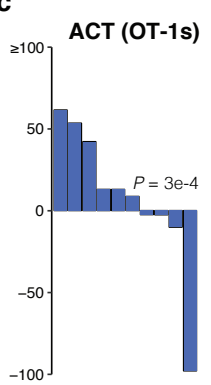

d

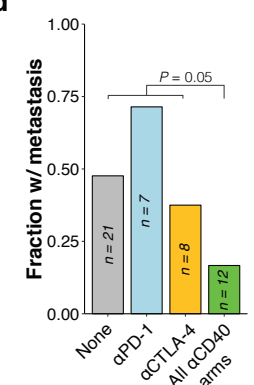


Extended Data Figure 5 Agonistic CD40 rescues deficient priming and enhances checkpoint blockade in low neoantigen-expressing tumors. (a) Schematic of preclinical trial design and dosing schedule. (b-c) Waterfall plots showing change in tumor size after 14 days of treatment, as determined by colonoscopy. ACT = adoptive cell transfer of OT-1s. (d) Fraction of mice with any metastases (liver, lung, or omentum), including only mice with progressive primary disease. Significance was assessed by $2 \times 2$ Fisher's exact test of fraction of mice with metastases in all aCD40 treatment arms (with and without ICB) versus all other arms (no treatment and ICB single agent arms). 


\section{Materials and methods}

Mice

Mice were housed in the animal facility at the Koch Institute for Integrative Cancer Research at MIT. All animal use was approved by the MIT Institutional Animal Care and Use Committee. Apc floxflox ${ }^{40}, K_{r a s}^{L S L-}$

G12D 41, Trp53 floxfllox 42, Rag2-/ 43, OT-144, and R26 Cas9-2A-EGFP 45 mice were maintained on a pure C57BL/6 background. Approximately equal numbers of male and female mice between 6 to 10 weeks of age were used for all experiments. Organoids were derived from female C57BL/6 mice, allowing transplant into male and female recipients without sex chromosome-specific immune responses. Io ${ }^{\text {SIIN }}$ organoids were transplanted into R26 Cas9-2A-EGFP mice, which are tolerant to EGFP, preventing T cell responses against EGFP. hiSIIN organoids were transplanted into wild-type or R26Cas9-2A-EGFP mice, with no differences in phenotype observed between genotypes.

\section{Lentivirus Production}

Lentivirus was produced in HEK-293 cells co-transfected with lentiviral backbone constructs, packaging vectors (psPax2 and pMD2G), and TransIT-LT1 Transfection Reagent (Mirus Bio). The HEK293s and transfection reagents were incubated at $37^{\circ} \mathrm{C}$ overnight. The following day, the culture media was changed. Virus was harvested 48 and 72 hours post transfection and filtered using a 0.45 micron Stericup Quick Release-HV Sterile Vacuum Filtration System (EMD Millipore). Virus was concentrated using an ultracentrifuge spinning at 25,000 rpm for 2 hours at $4^{\circ} \mathrm{C}$. Virus was resuspended in Opti-MEM (ThermoFisher).

\section{Organoid isolation and culture}

Normal colon crypts were isolated from wild-type female C57BL/6 mice as previously described ${ }^{13}$. Briefly, colons were isolated, rinsed in PBS, cut into small pieces and crypts dissociated with 5 mM EDTA at $4{ }^{\circ} \mathrm{C}$ for 1 
hr. Crypts and organoids were cultured in $65 \mu \mathrm{L}$ domes comprised of $10 \mu \mathrm{L}$ conditioned L-WRN (for primary crypts and WT organoids) or minimal media (for Apc knockdown organoids) and $55 \mu \mathrm{L}$ of growth factor reduced phenol-red free Matrigel Matrix (Corning) in 24-well TC treated Olympus plates (Genesee Scientific). Conditioned L-WRN media was produced as previously described ${ }^{46}$. Minimal media is comprised of $50 \mathrm{X}$ B-27 Serum-Free Supplement (Thermo), Penicillin and Streptomycin (Corning), and 100x L-Glutamine (Gibco). Organoids were passaged when confluent, every two to three days. To passage, Matrigel domes were mechanically dissociated in $500 \mu \mathrm{L}$ of TrypLE Express (Thermo) per well and by pipetting up and down in a 1 $\mathrm{mL}$ pipette 30 times before incubation at $37^{\circ} \mathrm{C}$ for 15 minutes. Following incubation, the organoids were pipetted another 30 times before being washed in PBS. Following centrifugation for 1 minute at 2000 rpm, cells were resuspended and plated as described above. Domes were allowed to solidify for 12 minutes in a $37^{\circ} \mathrm{C}$ incubator, and $500 \mu \mathrm{L}$ of appropriate media added.

\section{Organoid infection and transformation}

Confluent organoids were dissociated to single cells using Trype-LE Express, diluted and washed with PBS and then spun down in a tabletop centrifuge. They were then resuspended in $1.5 \mathrm{~mL}$ of appropriate media ( $\mathrm{L}-$ WRN media for primary lines, minimal media for transformed lines) with $10 \mu \mathrm{M}$ of $\mathrm{Y}-27632$ (Sigma-Aldrich).

Organoids were then divided into three separate wells of a 24-well TC-treated plate, and given a range of virus from $10 \mathrm{k}$ to $100 \mathrm{k}$ TU. The plate was then sealed using Parafilm, spun in a tabletop centrifuge at $600 \mathrm{~g}$ for 1 hour, and incubated for 5 hours at $37^{\circ} \mathrm{C}$, after which organoids were plated in Matrigel as described above. Adeno-Cre was used to recombine Kras ${ }^{L S L-G 12 D}$ and Trp53 floxfllox alleles in wild-type KP organoids, after which organoids were grown in L-WRN media + Y-27632 for 4 days, and then selected for recombination for 1 week using $10 \mu \mathrm{M}$ NUTLIN-3A (Sigma-Aldrich), which arrests growth of Trp53 wild-type organoids. Complete recombination of Kras and Trp53 alleles was confirmed by PCR allele-specific genotyping using published primers for these alleles ${ }^{41,42}$. Next, organoids were infected with shApc-expressing lentiviruses. To select for stable integration, organoids were switched to minimal media lacking WNT (described above) one week after infection, as organoids with Apc expression are unable to grow in the absence of WNT. Organoids with the 
lowest multiplicity of infection ( 0.5), as determined by mScarlet or GFP fluorescence, were chosen. Finally, these organoids were infected with integration-deficient lentivirus expressing Cas9 (lenti CRISPR v2) ${ }^{47}$ and sgRNA against Smad418, and selected by addition of TGF $\beta$, which arrests growth of Smad4-expressing organoids. Integration deficient lentivirus was generated using a D64V mutant psPax2 packaging vector ${ }^{48}$, and absence of integration in organoids was confirmed by absence of Cas9 protein by Western blot, and sensitivity to puromycin killing, following 1 week selection with TGF $\beta$.

Preparation of organoids for flow cytometry

Organoids were analyzed for expression of lentiviral constructs post-selection via flow cytometry. To dissociate organoids into single cells, Matrigel plugs were pipetted vigorously up and down 20 times in $400 \mu \mathrm{L}$ Dispase (Corning), and incubated at $37^{\circ} \mathrm{C}$ for 30 minutes. Following incubation, they were pipetted up and down 20 times, washed with $1 \mathrm{~mL}$ PBS, resuspended in $1 \mathrm{~mL}$ TrypLE Express, vigorously pipetted 20 times and incubated at $37^{\circ} \mathrm{C}$ for 12 minutes. Cells were pipetted up and down 20 times again and resuspended in 400 $\mu \mathrm{L}$ FACS buffer for analysis.

Preparation of organoids for injection

Intact organoids were harvested for injections two days after passaging. Organoids were washed in PBS and dissociated using Dispase (Corning) at a ratio of $100 \mu \mathrm{L}$ per $65 \mu$ l Matrigel dome. Matrigel was broken up by scraping and pipetting up and down four times using a $1 \mathrm{~mL}$ pipette and incubated at $37^{\circ} \mathrm{C}$ for 30 minutes. Organoids were then harvested into a $15 \mathrm{~mL}$ conical, washed with $10 \mathrm{~mL}$ PBS, and resuspended in OPTIMEM (Gibco). Whole organoids were counted and resuspended to a final concentration of 50,000 organoids / $\mathrm{mL}$ in OPTI-MEM and 10\% Matrigel. 
Lentiviral injection and orthotopic transplant of transformed organoids was performed following the methods described in Roper et al., 2018 ${ }^{13}$. First, mice were anesthetized using $2 \%$ isoflurane. Once anesthetized, mice were placed in a supine position and the colon was cleaned using a rapid enema of tap water. Gentle abdominal pressure was applied to propel stool out of the colon. Next the colonoscope was inserted and the colon insufflated with air. $100 \mu \mathrm{L}$ of lentivirus or organoids was taken up into the injection syringe (Hamilton Inc., part number 7656-01), which was then attached to the injection needle (Hamilton, 33 gauge, small Hub RN NDL, 16 inches long, point 4, 45-degree bevel, like part number 7803-05) which was fed through the working channel of the colonoscope. Next, the needle was inserted into the mucosa of the colon at around a $30^{\circ}$ angle relative to the colon wall. Next, a "test" injection of around $10 \mu \mathrm{L}$ was performed. If the "test" injection resulted in a small mucosal bubble, another $40 \mu \mathrm{L}$ was quickly injected. Successful injections result in large "blebs" within the mucosa. Lentivirus was injected at 20,000 or 100,000 TU / $\mu \mathrm{L}$. Organoids were injected at 50 intact organoids / $\mu \mathrm{L}$. Organoids were always prepared for injection at day 2 post-splitting.

Tissue preparation and flow cytometry

The colon draining lymph nodes (caudal and iliac) of each mouse were harvested into a single well of a 24 well TC plate (Corning) that had been scoured in a crosshatch pattern with a scalpel and containing $1 \mathrm{~mL}$ RPMI1640 (Corning) with 5\% heat-inactivated fetal bovine serum (HI-FBS) (harvest media). Next, the hardplastic end of a $3 \mathrm{~mL}$ syringe was used to grind the lymph nodes along the scoured plate. The cells were then collected and the well washed with an additional $1 \mathrm{~mL}$ of media. Lymph nodes were then washed with PBS and divided for immediate fluorescent antibody staining or for peptide stimulation.

Tumor regions were identified using a Dual Fluorescent Protein Flashlight, Model DFP-1 (Nightsea) and were harvested with approximately $1 / 4 \mathrm{~cm}$ sections of colon on either side. Tumors were then placed in a digestion buffer comprised of 1:104 Collagenase Type 1 (Worthington) and 1:500 DNAse (Sigma-Aldrich) in harvest media. Samples were then minced using surgical scissors before being digested at $37^{\circ} \mathrm{C}$ for 40 minutes with gentle agitation. Following digestion, samples were further dissociated with a gentleMACS Octo Dissociator 
(Miltenyi Biotec) on the tumor_imp1.1 setting. Cells were then filtered through a $100 \mu \mathrm{M}$ filter, washed in PBS, and divided for either immediate fluorescent antibody staining, or for functional analysis via peptide stimulation. During initial optimization of this protocol, intravenous CD45 staining, prior to sacrifice of animals, was performed to differentiate tissue-infiltrating versus circulating T cells. This resulted in staining of less than $1 \%$ of total SIINFEKL-specific T cells, and therefore was not routinely performed.

Prior to cell surface stains, live/dead staining was performed in PBS with ghost ef780 (Corning) at 1:500 for 15 minutes at room temperature. Next, cells were washed twice in FACS buffer (1 mM EDTA, 25 mM HEPES, $0.5 \% \mathrm{HI}-\mathrm{FBS}$ in PBS) and then stained using fluorescent antibodies for cell surface markers and SIINFEKL tetramer (NIH tetramer core) for 1 hour on ice. Cells were then fixed for 1 hour at room temperature in Fixation/Permeabilization Concentrate (Thermo Scientific) diluted 1:3 in Fixation/Permeabilization diluent (Thermo Scientific). Cells were then washed twice in permeabilization buffer (Thermo Scientific) and incubated for 15 minutes at room temperature. Intracellular staining was performed in permeabilization buffer overnight at $4{ }^{\circ} \mathrm{C}$. The following day, the cells were washed twice with FACS buffer and resuspended for analysis.

For flow cytometric analysis, single lymphocytes were gated first on FSC-A versus SSC-A and then FSC-A versus FSC-H. Then, live CD8 ${ }^{+} \mathrm{T}$ cells were gated on CD8a positivity and absence of ghost ef780 (Corning) live/dead staining. Antigen-specific CD8+ T cells were further gated on CD44 and SIINFEKL tetramer positivity. Expression of additional markers was analyzed specifically in this antigen-specific CD8 ${ }^{+} \mathrm{T}$ cell population in all flow cytometric experiments on T cells presented in this manuscript.

\section{Antigen-specific in vivo killing assay}

Splenocytes were prepared for in vivo transfer as described by Durward et. al, $2010^{26}$. Briefly, spleens were harvested from female C57BI/6 mice and mashed through a $70 \mu \mathrm{m}$ filter, red blood cells lysed using ACK lysing buffer (Thermo Fisher), and splenocytes resuspended in PBS in a round bottom 96-well plate at $1 \times 10^{8}$ cells per $\mathrm{mL}$. Half of the wells were pulsed with SIINFEKL peptide (Anaspec) at $1 \mu \mathrm{g} / \mathrm{mL}$, followed by labeling 
with the membrane dye CellTrace Violet (CTV; Thermo Fisher) at $20 \mu \mathrm{M}$. The remaining wells were labeled with $2 \mu \mathrm{M}$ CTV. Peptide loaded "target" and unloaded control splenocytes were then mixed 1:1 and $200 \mu \mathrm{L}$ retroorbitally injected $\left(2 \times 10^{7}\right.$ cells) into experimental animals 8 and 14 days post-transplant of organoids. DLNs and spleens were harvested 6 hours later and processed for flow cytometry as described above. Target and control splenocytes were identified by live/dead staining and CTV labeling intensity, and percent target killing determined relative to the control population. Targets killed per antigen-specific T cell was determined by dividing the total number of targets killed (control minus target splenocytes) by the total number of SIINFEKL tetramer ${ }^{+} \mathrm{CD}^{+} \mathrm{T}$ cells. This metric was meaningful at 14 days when target killing was incomplete in both lo $\mathrm{O}^{\mathrm{SIN}}$ and hisIIN animals, but precluded at 8 days by effectively complete target killing in hisIIN animals.

\section{Peptide stimulation for cytokine staining}

Samples were prepared as described above, and prior to surface staining were stained with antibodies for Ly6G (BioLegend, 1A8, 1:200), EpCAM (Biolegend, G8.8 1:80) and F4/80 (Biolegend, BM8, 1:80) in FACS buffer for 30 minutes at $4{ }^{\circ} \mathrm{C}$ and then depleted using Dynabeads Goat Anti-mouse IgG kit (Thermo Fisher) following manufacturer recommendations. T cells were then stimulated in T-cell media (RPMI with 10\% HI-FBS, 20 mM HEPES, 1 mM Sodium Pyruvate, 2 mM L-Glutamine, $50 \mu \mathrm{M} \beta$-mercaptoethanol, 1x non-essential amino acids, and 0.5x Penicillin/Streptomycin) with 1:1000 GolgiPlug (BD) and $2 \mu \mathrm{M}$ Monensin Solution (BioLegend), and 1 $\mu \mathrm{M}$ SIINFEKL peptide (Anaspec) for 3 hours at $37^{\circ} \mathrm{C}$. Cells were washed twice in PBS and stained for surface and intracellular markers following the procedure described above.

\section{OT-1 T cell activation}

Spleens and LNs from OT-1 mice were harvested into ice cold PBS and mashed through a $100 \mu \mathrm{M}$ filter using the rubber end of a syringe plunger. Cells were pelleted, red blood cells lysed using ACK Lysing Buffer (Gibco), resuspended in T-cell media (described in previous section) $+10 \mathrm{ng} / \mathrm{mL}$ hlL2 (PeproTech) and $1 \mu \mathrm{M}$ SIINFEKL peptide (Anaspec), counted, and plated in a 24 well TC plate at $1 \times 10^{6} \mathrm{cells} / \mathrm{mL}, 1.5 \mathrm{~mL}$ per well. 
Stimulation was performed for 24 hours at $37^{\circ} \mathrm{C}$. CD8 $+\mathrm{T}$ cells were then purified using the CD8a+ T Cell Isolation Kit, mouse (Miltenyi Biotech), and expanded in T cell media + hIL2 with daily splitting. T cells were used for ACT or co-culture assays at day 3 or 4.

\section{OT-1 and organoid co-culture}

OT-1 T cells were activated in vitro as described above. Organoids were broken up into single cells as described in the organoid infection section. Organoids and OT-1s were counted with a hemocytometer and plated at 0:1 and 5:1 effector to target ratios (effector $=$ OT-1s, target = organoid cells) at 2500 organoid single cells in $10 \mu \mathrm{l}$ minimal media and $50 \mu \mathrm{l}$ Matrigel. Co-cultures were plated in triplicate at $20 \mu \mathrm{l}$ per dome, grown in minimal media, and imaged on day 4. Total fluorescent area of organoids within images was quantified in ImageJ by setting left and right thresholds of greyscale images to 22 and 255 on B\&W setting, respectively, and analyzing particles with size threshold set to $>20$ pixels ${ }^{2}$, circularity set to $0.1-1.0$, and including holes. Areas of objects were summed and plotted in R.

\section{Three color immunohistochemistry}

Tissue samples were fixed in zinc formalin overnight and washed in $70 \%$ ethanol before embedding in paraffin. Tissue slides were dewaxed using a Gemini automatic self-contained slide strainer. Next, slides underwent antigen retrieval through incubation in citrate buffer $\mathrm{pH} 6$ in a pressure cooker set at $125^{\circ} \mathrm{C}$ for five minutes. Endogenous enzyme activity was blocked using BLOXALL Endogenous Peroxidase and Alkaline Phosphatase Blocking Solution (Vector). Endogenous immunoglobulins were blocked using R.T.U Normal Horse Serum (2.5\%) (Vector). Slides were stained with CD8a (ab217344, Abcam) 1:1000 overnight. The following day, the slides were incubated with Alkaline Phosphatase (AP) anti-Rabbit IgG (Vector) and developed using the Vector Black substrate (Vector). Next, sections underwent a second round of antigen retrieval in a pressure cooker set at $110{ }^{\circ} \mathrm{C}$ for two minutes. Sections were subsequently co-stained with FOXP3 (FJK-16s, eBioscience) 1:125 and CD4 (ab183685, Abcam) 1:400. Sections were then incubated first with AP anti-Rat IgG (Vector), and then 
HRP anti-Rabbit IgG (Vector). FOXP3 staining was developed using Vector Red (Vector) first, followed by CD4 staining using Vina Green (Biocare Medical). Slides were counterstained with Harris Acidified Hematoxylin and dehydrated in a Gemini automatic self-contained slide stainer. Aqueous wash steps following counterstain were shortened from 1 minute to 30 seconds to minimize loss of Vina Green stain.

Immune infiltration was calculated by a convolutional neural network (CNN) trained to identify the three cell types stained $($ black $=$ CD8, green $=$ CD4, green/red $=$ Treg), using Aiforia's cloud-based platform (Aiforia Technologies Oy). Whole slides were scanned with a Leica AT2 (Aperio) using the Rainbow color profile. First, the CNN was trained to identify a tissue layer. Within that layer, the CNN was trained to identify separate layers containing black, green, and green/red staining. Within each of these layers, an object counter was trained to quantify the number of cells with the particular stain. Training was performed by manual annotation of layers and counting of objects within training regions. The algorithm was trained on 20 separate slides, with roughly five training regions per layer per slide. Performance was validated against human counting and found to be highly accurate and consistent.

In situ SIINFEKL tetramer staining and immunofluorescent imaging

Tissue was stained in situ with SIINFEKL tetramer as previously described ${ }^{49}$. Briefly, freshly harvested tissue was manually cut into thin sections with a razor blade and incubated with PE-conjugated SIIINFEKL tetramer (NIH tetramer core) diluted to $2 \mu \mathrm{g} / \mathrm{mL}$ in PBS with $2 \%$ HI-FBS overnight at $4{ }^{\circ} \mathrm{C}$. The next day, tissue was washed and lightly fixed in $2 \%$ PFA for 2 hours on ice. Tissue was then washed again and incubated overnight in $30 \%$ sucrose at $4{ }^{\circ} \mathrm{C}$. The next day, tissue was snap frozen in Tissue Tek OCT (Sakura) using a 2methylbutane cooling bath in a beaker on dry ice. Sections were cut to a thickness of $7 \mu \mathrm{m}$ on a CryoStar NX70 (ThermoFisher), fixed in acetone at $-20{ }^{\circ} \mathrm{C}$ for 10 minutes, and stored at $-20{ }^{\circ} \mathrm{C}$.

Prior to additional staining, slides were rehydrated in PBS for 10 minutes, and then blocked with $5 \%$ bovine serum albumin in PBS for 45 minutes in a humidified chamber. CD8ß AF647 (YTS156.7.7, BioLegend) (1:100), 
and anti-human $\beta 2$-microglobulin ( $\beta 2 \mathrm{M})$ PE (2M2, BioLegend) (1:50) were added in a total volume of $300 \mu \mathrm{L}$ of blocking solution and incubated at $4^{\circ} \mathrm{C}$ overnight. Anti- $\beta 2 \mathrm{M}$ staining is specific to human $\beta 2 \mathrm{M}$ in the SIINFEKL tetramer and serves to amplify signal. 30 minutes prior to imaging, $120 \mu \mathrm{L}$ of ProLong Diamond Antifade Mountant with DAPI (ThermoFisher) was added to slides. Images were taken at 30X on an Olympus FV1200 Laser Scanning Confocal Microscope and analyzed in ImageJ.

In vivo antibody dosing

All antibody dosing was performed via intraperitoneal injection using a $1 \mathrm{~mL}$ syringe and a 32 -gauge needle in PBS. aCD4 (GK1.5, BioXCell) and aCD8 (2.43, BioXCell) depleting antibodies were administered at $200 \mathrm{ug}$ every 4 days. aPD-1 (29F.1A12, BioXCell) was administered at $200 \mu \mathrm{g}$ three times a week. aCTLA (9H10, BioXCell) was administered at an initial dose of $200 \mu \mathrm{g}$, with all subsequent doses at $100 \mu \mathrm{g}$, three times a week. aCD40 (FGK4.5, BioXCell) was administered once at the beginning of treatment at $100 \mu \mathrm{g}$.

Therapeutic vaccination

The adjuvant amphiphile-CpG (amph-CpG) and antigen amphiphile (amph-peptide) were produced as previously described $^{50}$. Briefly, class B CpG 1826 oligonucleotide with a $\mathrm{G}_{2}$ spacer (5'-diacyl lipidGGTCCATGACGTTCCTGACGTT- 3') was conjugated via the 5' end to an 18 carbon diacyl tail. Antigen peptide $\mathrm{OVA}_{250-270}$ (CGLEQLESIINFEKLTEWTSS) and non-specific mutant gp100 $20-39$ (optimized S27P, EGP long51, CAVGALEGPRNQDWLGVPRQL) were conjugated via their N'cysteine residue to 1,2-distearoyl-sn-glycero-3phosphoethanolamine- $N$-[maleimide(polyethyleneglycol-2000] (Avanti Polar Lipids). Mice were vaccinated subcutaneously at the base of the tail with $1.24 \mathrm{nmol}$ amph-CpG and $25 \mu \mathrm{g}$ of amph-peptide, with half of the dose given to each side. Vaccination was performed once weekly, starting 14 days post-transplant of losilN organoids. 
Tumor progression was monitored longitudinally using a Karl Storz colonoscopy system with white light, RFP and GFP fluorescence. This consists of Image 1 H3-Z Spies HD Camera System (part TH100), Image 1 HUB CCU (parts TC200, TC300), 175 Watt D-Light Cold Light Source (part 20133701-1), AIDA HD capture system, and fluorescent filters in the RFP and GFP channels (all from Karl Storz). The endoscope used for imaging was the Hopkins Telescope (Karl Storz, part 64301AA) with operating sheath (Karl Storz, part 64301AA). To consistently measure tumor area, biopsy forceps (Richard Wolf) were fed through the operating sheath and positioned as consistently as possible given two major landmarks: widthwise grooves that appear as concentric semi-circles in the field of view, and a lengthwise groove at the forceps tip. Images were captured upon contact of the forceps with the tumor to maintain consistent distance between camera and tumor across all images. Tumor area in the field of view was calculated using ImageJ. Imaging and quantification were performed blind to treatment groups.

\section{TCGA and neoantigen prediction data analysis}

Colon and rectal adenocarcinoma (COADREAD) RNA-seq Level 2 normalized data and patient information was downloaded from the $\mathrm{NCl}$ Genomics Data Commons data portal using the 'TCGAbiolinks' package in $\mathrm{R}^{52}$. Normal tissue, FFPE-fixed samples, and other duplicates were removed prior to analysis. Predicted neoantigens and affinities were retrieved from a previously published pan-cancer analysis ${ }^{53}$.

\section{TCR sequencing}

SIINFEKL tetramer-positive CD8 ${ }^{+}$T cells were prepared and stained as described for single cell RNAseq. Cells were directly sorted into $50 \mu$ lysis buffer with proteinase $\mathrm{K}$, from the Arcturus PicoPure DNA Extraction kit (ThermoFisher), in low binding microcentrifuge tubes (Biotix), and genomic DNA extraction performed following manufacturer instructions. Mouse TCR $\beta$ sequencing was performed by Adaptive Biotechnologies. Analysis was performed in $\mathrm{R}$, and Simpson diversity calculated using the 'vegan' package. To account for differences in 
total numbers of T cells surveyed in samples between groups, unique productive TCR sequences (clones) were randomly down-sampled to match between groups. Down-sampled data is presented in Extended Data Figure 3d-e, although down-sampling did not impact observed trends.

\section{Statistical Analysis}

Statistical analysis was performed in R. For statistical assessment of differences in proportionality, Fisher's exact $2 \times 2$ test was performed. For continuous data, Wilcoxon rank sum test was performed, with the exception of the organoid and OT-1 co-culture results, which were analyzed with the Student's T test. Multiple comparison correction was performed using Holm's method.

Flow cytometry antibodies

\begin{tabular}{|l|l|l|l|l|}
\hline Target & Fluorochrome & Dilution & Clone & Manufacturer \\
\hline TNFa & PE & 1 to 400 & MP6-XT22 & BioLegend \\
\hline GZMB & PE-CF594 & 1 to 250 & GB11 & BioLegend \\
\hline IFNY & PerCP-Cy5.5 & 1 to 200 & XMG1.2 & BioLegend \\
\hline PD-1 & BV421 & 1 to 400 & RMP-30 & BioLegend \\
\hline Ki67 & BV510 & 1 to 400 & B56 & BD Biosciences \\
\hline TIM3 & BV605 & 1 to 200 & RMT3-23 & BioLegend \\
\hline CD44 & BV711 & 1 to 200 & IM7 & BioLegend \\
\hline CD44 & BUV661 & 1 to 400 & IM7 & BD Biosciences \\
\hline Live/Dead & APC-ef780 & 1 to 500 & Ghost Dye & TONBO \\
\hline CD8a & BUV395 & 1 to 400 & $53-6.7$ & BioLegend \\
\hline LAG3 & BUV737 & 1 to 100 & C9B7W & BD Biosciences \\
\hline SIINFEKL Tetramer & PE & 1 to 400 & & NIH Tetramer Core \\
\hline
\end{tabular}




\begin{tabular}{|l|l|l|l|l|}
\hline 2B4 & PE-Cy7 & 1 to 200 & eBio244F4 & eBioscience \\
\hline TCF-1 & AF647 & 1 to 250 & C63D9 & Cell Signaling Technologies \\
\hline TIGIT & FITC & 1 to 100 & GIGD7 & eBioscience \\
\hline
\end{tabular}




\section{References}

1. Muzny, D. M. et al. Comprehensive molecular characterization of human colon and rectal cancer. Nature 487, 330-337 (2012).

2. Le, D. T. et al. Mismatch repair deficiency predicts response of solid tumors to PD-1 blockade. Science 357, 409-413 (2017).

3. Germano, G. et al. Inactivation of DNA repair triggers neoantigen generation and impairs tumour growth. Nature 552, 116-120 (2017).

4. Mandal, R. et al. Genetic diversity of tumors with mismatch repair deficiency influences anti-PD-1 immunotherapy response. Science 364, 485-491 (2019).

5. Samstein, R. M. et al. Tumor mutational load predicts survival after immunotherapy across multiple cancer types. Nat. Genet. 51, 202-206 (2019).

6. Karandikar, S. H. et al. Identification of epitopes in ovalbumin that provide insights for cancer neoepitopes. JCI Insight 4, (2019).

7. Lawrence, M. S. et al. Mutational heterogeneity in cancer and the search for new cancer-associated genes. Nature 499, 214-218 (2013).

8. Supek, F. \& Lehner, B. Differential DNA mismatch repair underlies mutation rate variation across the human genome. Nature 521, 81-84 (2015).

9. Steimle, A. \& Frick, J.-S. Molecular Mechanisms of Induction of Tolerant and Tolerogenic Intestinal Dendritic Cells in Mice. J. Immunol. Res. 2016, 1958650 (2016).

10. Kim, M. et al. Critical Role for the Microbiota in CX3CR1+ Intestinal Mononuclear Phagocyte Regulation of Intestinal T Cell Responses. Immunity 49, 151-163.e5 (2018).

11. Spranger, S., Bao, R. \& Gajewski, T. F. Melanoma-intrinsic $\beta$-catenin signalling prevents anti-tumour immunity. Nature 523, 231-235 (2015).

12. Galarreta, M. R. de et al. $\beta$-catenin activation promotes immune escape and resistance to anti-PD-1 therapy in hepatocellular carcinoma. Cancer Discov. (2019) doi:10.1158/2159-8290.CD-19-0074.

13. Roper, J. et al. In vivo genome editing and organoid transplantation models of colorectal cancer and metastasis. Nat. Biotechnol. 35, 569-576 (2017). 
14. DuPage, M. et al. Endogenous T cell responses to antigens expressed in lung adenocarcinomas delay malignant tumor progression. Cancer Cell 19, 72-85 (2011).

15. DuPage, M., Mazumdar, C., Schmidt, L. M., Cheung, A. F. \& Jacks, T. Expression of tumour-specific antigens underlies cancer immunoediting. Nature 482, 405-9 (2012).

16. Dow, L. E. et al. Apc Restoration Promotes Cellular Differentiation and Reestablishes Crypt Homeostasis in Colorectal Cancer. Cell 161, 1539-1552 (2015).

17. Drost, J. et al. Sequential cancer mutations in cultured human intestinal stem cells. Nature 521, 43-47 (2015).

18. de Sousa e Melo, F. et al. A distinct role for Lgr5+ stem cells in primary and metastatic colon cancer. Nature 543, 676-680 (2017).

19. Schell, M. J. et al. A multigene mutation classification of 468 colorectal cancers reveals a prognostic role for APC. Nat. Commun. 7, 11743 (2016).

20. Gubin, M. M. et al. Checkpoint blockade cancer immunotherapy targets tumour-specific mutant antigens. Nature 515, 577-81 (2014).

21. Mlecnik, B. et al. Integrative Analyses of Colorectal Cancer Show Immunoscore Is a Stronger Predictor of Patient Survival Than Microsatellite Instability. Immunity 44, 698-711 (2016).

22. Parish, I. A. et al. The molecular signature of CD8+ T cells undergoing deletional tolerance. Blood 113, 4575-85 (2009).

23. Lin, W.-H. W. et al. CD8+ T Lymphocyte Self-Renewal during Effector Cell Determination. Cell Rep. 17, 1773-1782 (2016).

24. Danilo, M., Chennupati, V., Silva, J. G., Siegert, S. \& Held, W. Suppression of Tcf1 by Inflammatory Cytokines Facilitates Effector CD8 T Cell Differentiation. Cell Rep. 22, 2107-2117 (2018).

25. Zhou, X. et al. Differentiation and persistence of memory CD8(+) T cells depend on T cell factor 1. Immunity 33, 229-40 (2010).

26. Durward, M., Harms, J. \& Splitter, G. Antigen specific in vivo killing assay using CFSE labeled target cells. J. Vis. Exp. 45 (2010) doi:10.3791/2250.

27. Wherry, E. J. T cell exhaustion. Nat. Immunol. 12, 492-499 (2011). 
28. McLane, L. M., Abdel-Hakeem, M. S. \& Wherry, E. J. CD8 T Cell Exhaustion During Chronic Viral Infection and Cancer. Annu. Rev. Immunol. 37, 457-495 (2019).

29. Miller, B. C. et al. Subsets of exhausted CD8+ T cells differentially mediate tumor control and respond to checkpoint blockade. Nat. Immunol. 20, 326-336 (2019).

30. Siddiqui, I. et al. Intratumoral Tcf1+PD-1+CD8+ T Cells with Stem-like Properties Promote Tumor Control in Response to Vaccination and Checkpoint Blockade Immunotherapy. Immunity 50, 195211.e10 (2019).

31. Kurtulus, S. et al. Checkpoint Blockade Immunotherapy Induces Dynamic Changes in PD-1-CD8+ Tumor-Infiltrating T Cells. Immunity 50, 181-194.e6 (2019).

32. Provine, N. M. et al. Immediate Dysfunction of Vaccine-Elicited CD8+ T Cells Primed in the Absence of CD4+ T Cells. J. Immunol. 197, 1809-22 (2016).

33. Cella, M. et al. Ligation of CD40 on dendritic cells triggers production of high levels of interleukin-12 and enhances T cell stimulatory capacity: T-T help via APC activation. J. Exp. Med. 184, 747-752 (1996).

34. Byrne, K. T. \& Vonderheide, R. H. CD40 Stimulation Obviates Innate Sensors and Drives T Cell Immunity in Cancer. Cell Rep. 15, 2719-2732 (2016).

35. O'Hara, M. H. et al. Abstract CT004: A Phase lb study of CD40 agonistic monoclonal antibody APX005M together with gemcitabine (Gem) and nab-paclitaxel (NP) with or without nivolumab (Nivo) in untreated metastatic ductal pancreatic adenocarcinoma (PDAC) patients. in Clinical Trials vol. 79 CT004-CT004 (American Association for Cancer Research, 2019).

36. Kabacaoglu, D., Ciecielski, K. J., Ruess, D. A. \& Algül, H. Immune Checkpoint Inhibition for Pancreatic Ductal Adenocarcinoma: Current Limitations and Future Options. Front. Immunol. 9, 1878 (2018).

37. Spiotto, M. T. et al. Increasing tumor antigen expression overcomes \&quot;ignorance\&quot; to solid tumors via crosspresentation by bone marrow-derived stromal cells. Immunity 17, 737-47 (2002).

38. Engels, B. et al. Relapse or eradication of cancer is predicted by peptide-major histocompatibility complex affinity. Cancer Cell 23, 516-26 (2013).

39. Schietinger, A. et al. Tumor-Specific T Cell Dysfunction Is a Dynamic Antigen-Driven Differentiation Program Initiated Early during Tumorigenesis. Immunity 45, 389-401 (2016). 
40. Kuraguchi, M. et al. Adenomatous Polyposis Coli (APC) Is Required for Normal Development of Skin and Thymus. PLoS Genet. 2, e146 (2006).

41. Johnson, L. et al. Somatic activation of the K-ras oncogene causes early onset lung cancer in mice. Nature 410, 1111-6 (2001).

42. Marino, S., Vooijs, M., van Der Gulden, H., Jonkers, J. \& Berns, A. Induction of medulloblastomas in p53-null mutant mice by somatic inactivation of $\mathrm{Rb}$ in the external granular layer cells of the cerebellum. Genes Dev. 14, 994-1004 (2000).

43. Hao, Z. \& Rajewsky, K. Homeostasis of peripheral B cells in the absence of B cell influx from the bone marrow. J. Exp. Med. 194, 1151-64 (2001).

44. Hogquist, K. A. et al. T cell receptor antagonist peptides induce positive selection. Cell 76, 17-27 (1994).

45. Chu, V. T. et al. Efficient generation of Rosa26 knock-in mice using CRISPR/Cas9 in C57BL/6 zygotes. BMC Biotechnol. 16, 4 (2016).

46. Miyoshi, H. \& Stappenbeck, T. S. In vitro expansion and genetic modification of gastrointestinal stem cells in spheroid culture. Nat. Protoc. 8, 2471-82 (2013).

47. Sanjana, N. E., Shalem, O. \& Zhang, F. Improved vectors and genome-wide libraries for CRISPR screening. Nat. Methods 11, 783-784 (2014).

48. Certo, M. T. et al. Tracking genome engineering outcome at individual DNA breakpoints. Nat. Methods 8, 671-676 (2011).

49. Steinert, E. M. et al. Quantifying Memory CD8 T Cells Reveals Regionalization of Immunosurveillance. Cell 161, 737-749 (2015).

50. Liu, H. et al. Structure-based programming of lymph-node targeting in molecular vaccines. Nature 507, 519-522 (2014).

51. Van Stipdonk, M. J. B. et al. Design of agonistic altered peptides for the robust induction of CTL directed towards $\mathrm{H}-2 \mathrm{Db}$ in complex with the melanoma-associated epitope gp100. Cancer Res. 69, 7784-7792 (2009).

52. Colaprico, A. et al. TCGAbiolinks: an R/Bioconductor package for integrative analysis of TCGA data. 
Nucleic Acids Res. 44, e71-e71 (2016).

53. Rooney, M. S., Shukla, S. A., Wu, C. J., Getz, G. \& Hacohen, N. Molecular and Genetic Properties of Tumors Associated with Local Immune Cytolytic Activity. Cell 160, 48-61 (2015).

\section{Acknowledgements}

This work was supported by the NCI Cancer Center Support Grant P30-CA14051, R01 CA233983 and the Howard Hughes Medical Institute. P.M.K.W was supported by a Damon Runyon Fellowship Award. We thank K. Yee, J. Teixeira, K. Anderson, and M. Magendantz for administrative support, and our colleagues in the Jacks laboratory and the broader community at the Koch Institute of MIT for thoughtful discussions and technical advice, with specific thanks to Dr. Stefani Spranger and members of her laboratory. We also thank the Koch Institute Swanson Biotechnology Center for core support from the Flow Cytometry, Histology, and Microscopy facilities, and T. Westerling and Aiforia for help developing an automated CNN for IHC quantification. Finally, we thank T. Tammela and J. Roper for early inspiration and mentorship in the colonoscopy-guided injection technique.

\section{Author contributions}

P.M.K.W and T.J conceived and directed the study. P.M.K.W, N.S., O.S., and H.H. carried out all aspects of the research, animal care and experimentation. J.M.S provided essential conceptual and technical guidance in the design and execution of flow cytometry-based experiments. N.S. developed the triple IHC and an automated CNN for quantification in collaboration with Aiforia. N.S., O.S., D.Z., J.J.P, and M.C.B generated lentiviral constructs and primary organoid lines used in the study. C.M.B. and D.J.I. provided guidance, reagents, and technical assistance with therapeutic vaccinations. G.E and O.Y. provided important guidance and reagents for organoid culture and colonoscopy-guided injections. Data analysis was carried out by P.M.K.W. The manuscript was written by P.M.K.W and T.J. with feedback from all authors. 


\section{Competing interest declaration}

T.J. is a member of the Board of Directors of Amgen and Thermo Fisher Scientific, and a co-founder of

Dragonfly Therapeutics and T2 Biosystems. T.J. serves on the Scientific Advisory Board of Dragonfly

Therapeutics, SQZ Biotech, and Skyhawk Therapeutics. The Jacks laboratory also currently receives funding from Johnson \& Johnson, but this did not support the research described in this manuscript.

\section{Data availability}

All appropriate data will be made publicly available upon acceptance for publication.

\section{Code availability}

All appropriate code will be made publicly available upon acceptance for publication.

\section{Additional Information}

Supplementary Information is available for this paper. Correspondence and requests for materials should be addressed to Tyler Jacks (tjacks@mit.edu). 


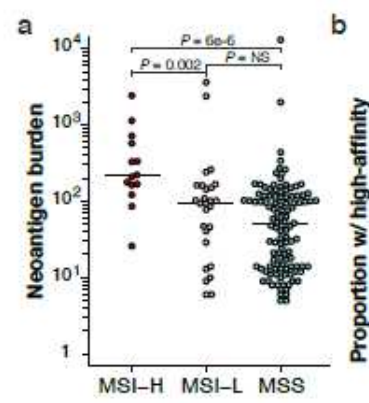

e

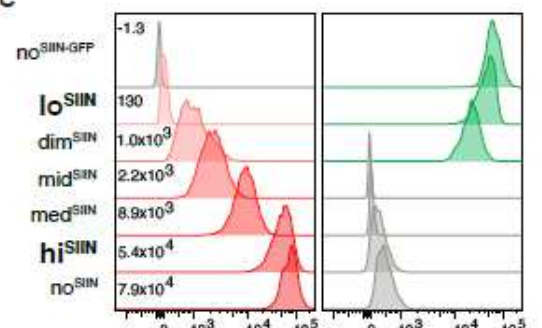

j

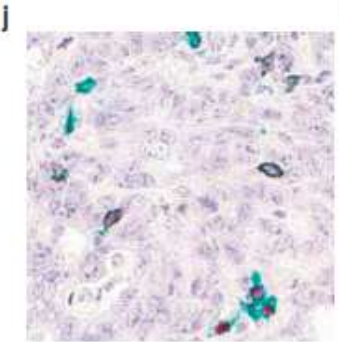

m

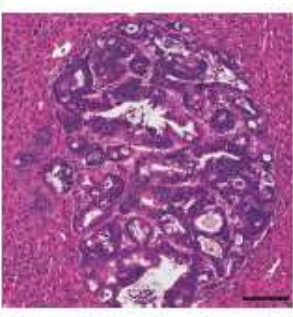

$c$

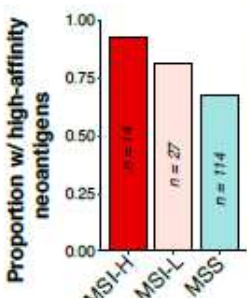

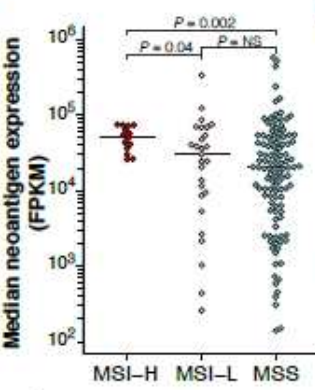

$f$

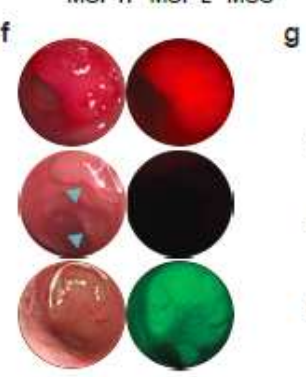

k

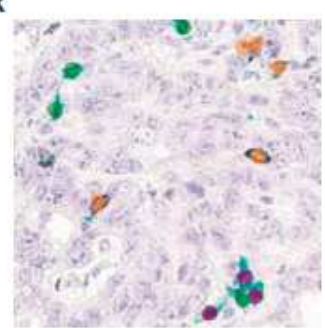

n

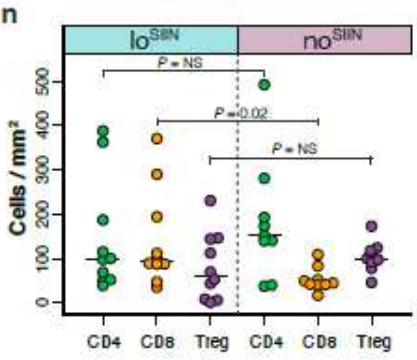

d no $^{\text {SIIN }}$

I
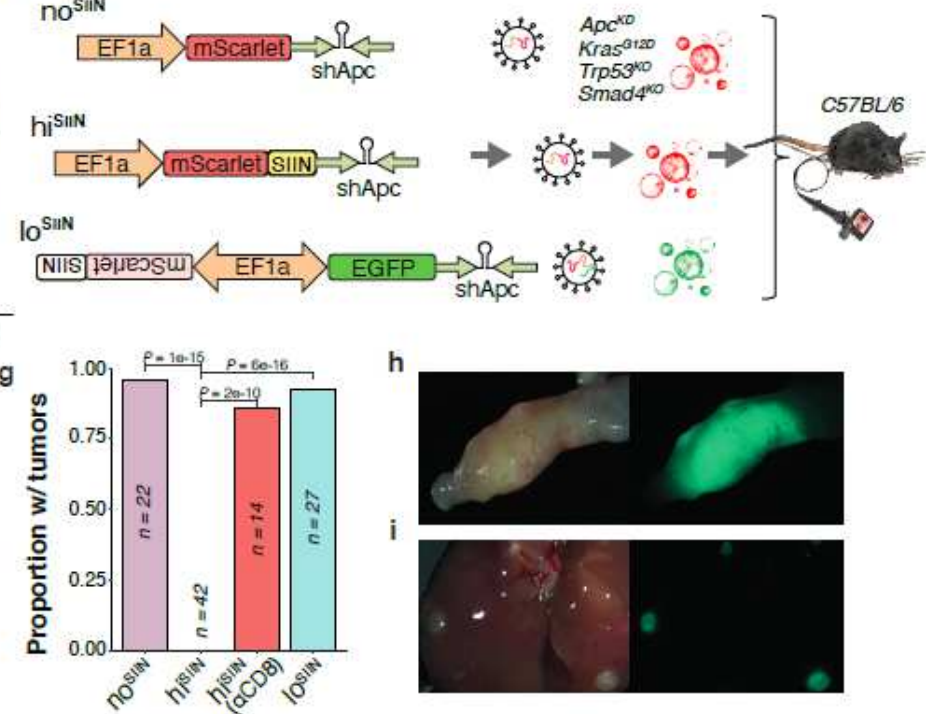

h
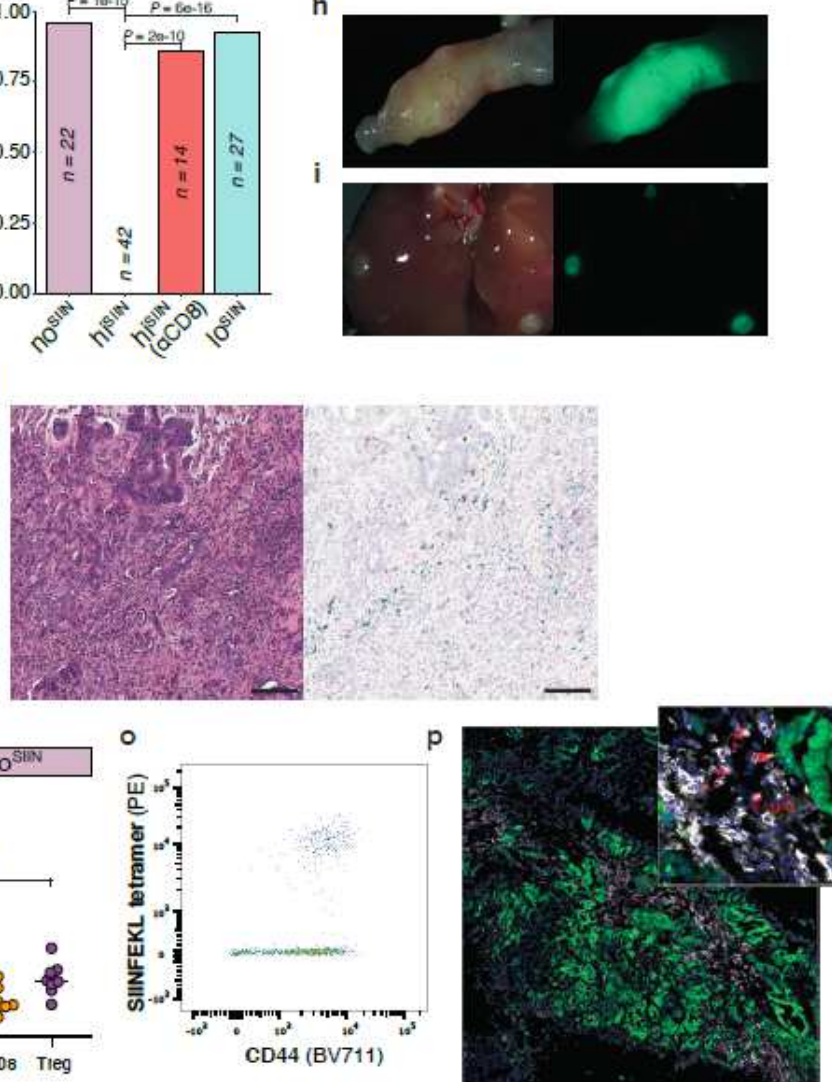

\section{Figure 1}

A novel model of $\mathrm{CRC}$ reveals a critical role of neoantigen expression level in tumor immune evasion. (ac) Analysis of predicted neoantigens in human CRC (TCGA COADREAD) with high MSI (MSI-H), low MSI (MSI-L), and MSS. (a) Total expressed neoantigens with strong predicted HLA binding (IC50 $\leq 500 \mathrm{nM}$ ) by patient. (b) Proportion of patients expressing at least one neoantigen with predicted binding affinity equal to or greater than SIINFEKL (IC50 $\leq 10 \mathrm{nM})$. (c) Median expression of neoantigens by patient (FPKM, upper quartile-normalized). (d) shApc-expressing lentiviruses used to transform KP organoids, with no (noSIIN), high (hiSIIN) and low (IoSIIN) expression of SIINFEKL. Resulting shAKPS organoids were orthotopically-transplanted into the colons of syngeneic mice. (e) Expression of mScarlet/mScarletSIIN and EGFP in expression variant organoids by flow cytometry. (f) Colonoscopy images of noSIIN tumor (RFP channel), hiSIIN scars (RFP channel, blue arrows indicate injection sites), and loSIIN tumor (GFP channel) 28 days post-transplant. (g) Efficiency of tumor formation 42 days post-transplant with noSIIN, 
hiSIIN, hiSIIN with antibody depletion of CD8+ T cells, and IoSIIN organoids. (h-i) Stereoscopic brightfield and fluorescent images of loSIIN colon tumor (h) and liver metastases (i) 42 days post-transplant. (j-k) Example three color IHC (black $=\mathrm{CD} 8$, green $=\mathrm{CD} 4$, red $=$ FOXP3) $(\mathrm{J})$ and automated annotation by convolutional neural network (k). (I-m) Representative H\&E and three color IHC of loSIIN primary colon tumor (I) and liver metastasis (m) 42 days post-transplant. Scale bar $=100 \mu \mathrm{M}$. (n) Quantification of CD8, CD4, and Treg cells infiltrating loSIIN and noSIIN tumors by convoluted neural network analysis. Each point represents at least one tumor from a single animal. (o-p) Identification of SIINFEKL tetramer-specific CD8+ T cells infiltrating 42 day loSIIN tumors by flow cytometry (o) and immunofluorescence $(p)$ with in situ tetramer staining (green $=$ tumor, white $=\mathrm{CD} 8$, red $=$ SIINFEKL tetramer, blue $=\mathrm{DAPI}$ ).

a
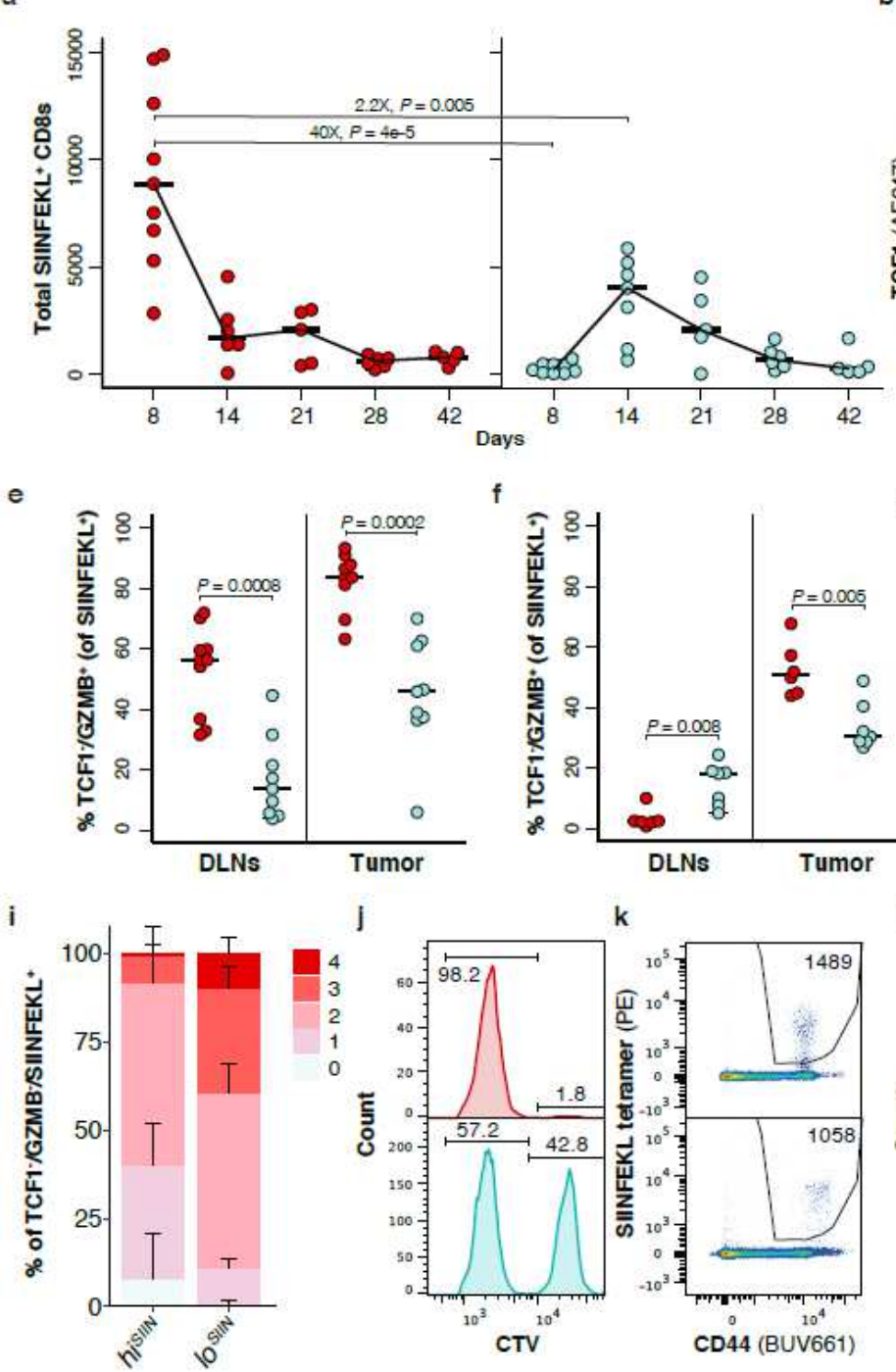

j

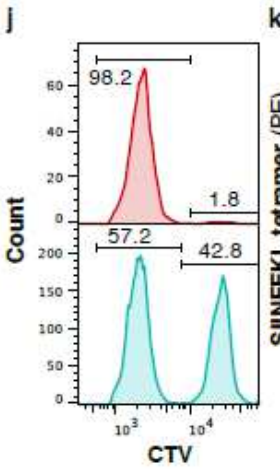

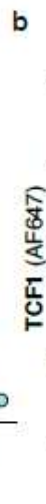

f

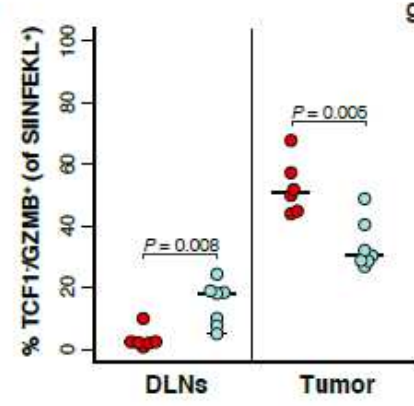

k

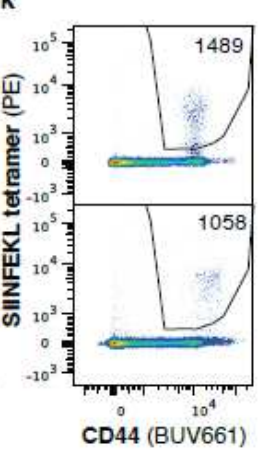

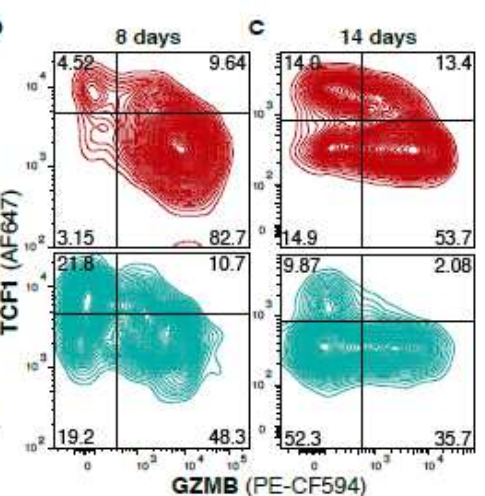

d

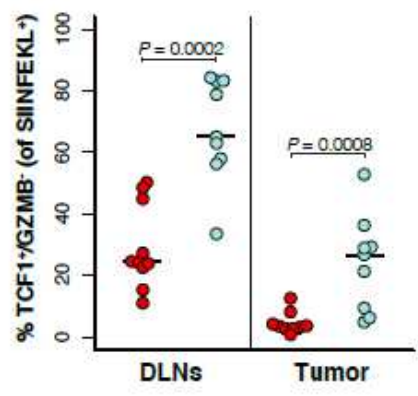

g
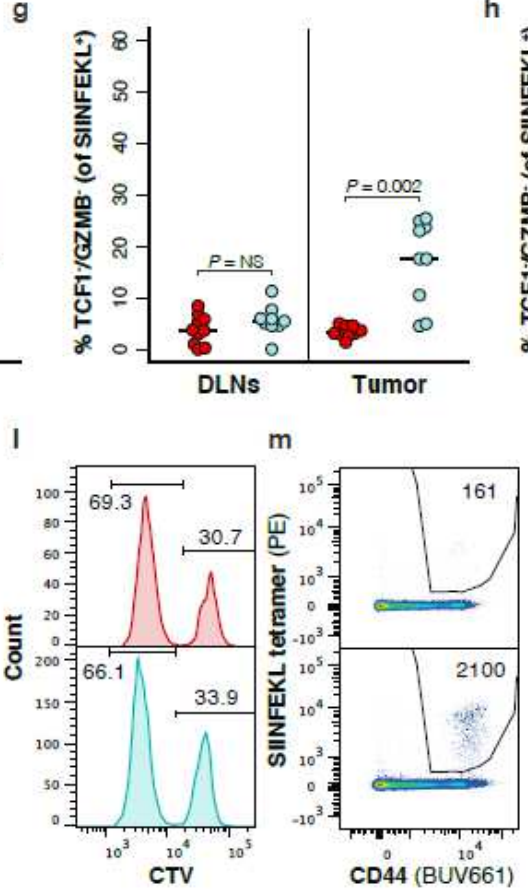

h
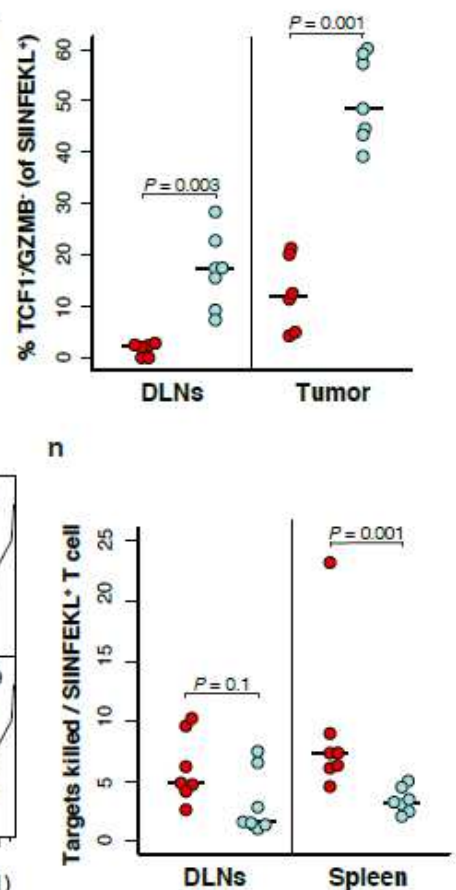

Figure 2

Low neoantigen expression drives impaired $T$ cell effector commitment and immediate dysfunction. (a) Total numbers of CD44+/CD8+ antigen-specific $T$ cells isolated from tumors at 8, 14, 21, 28, and 42 days post-transplant of hiSIIN (red) and loSIIN (blue) organoids by flow cytometry. (b-c) Antigen-specific T cell expression of TCF1 versus GZMB in tumors at 8 (b) and 14 (c) days. (d-e,g) Percent of antigen-specific T cells from DLNs and tumors at 8 days positive for TCF1 and negative for GZMB (d), negative for TCF1 
and positive for GZMB (e), and double-negative for TCF1 and GZMB (g). (f,h) Percent of antigen-specific T cells from DLNs and tumors at 14 days negative for TCF1 and positive for GZMB (f), and doublenegative for TCF1 and GZMB (h). (i) Percentage of TCF1-/GZMB- antigen-specific T cells from tumors at 14 days expressing $0,1,2,3$, and 4 inhibitory receptors (PD-1, TIM3, LAG3, and 2B4). (j-m) Representative in vivo killing assay histograms of transferred control (weak CTV stain) and SIINFEKL-loaded "target" (strong CTV stain) splenocytes and flow plots of antigen-specific T cells recovered from DLNs at 8 (j-k) and 14 (I-m) days post-transplant of hiSIIN (red) and loSIIN (blue) organoids. Percent of control and target populations are noted in histograms, and total number of antigen-specific T cells noted in flow plots. (n) Target killing normalized to number of antigen-specific T cells recovered in 14 day in vivo killing assay.
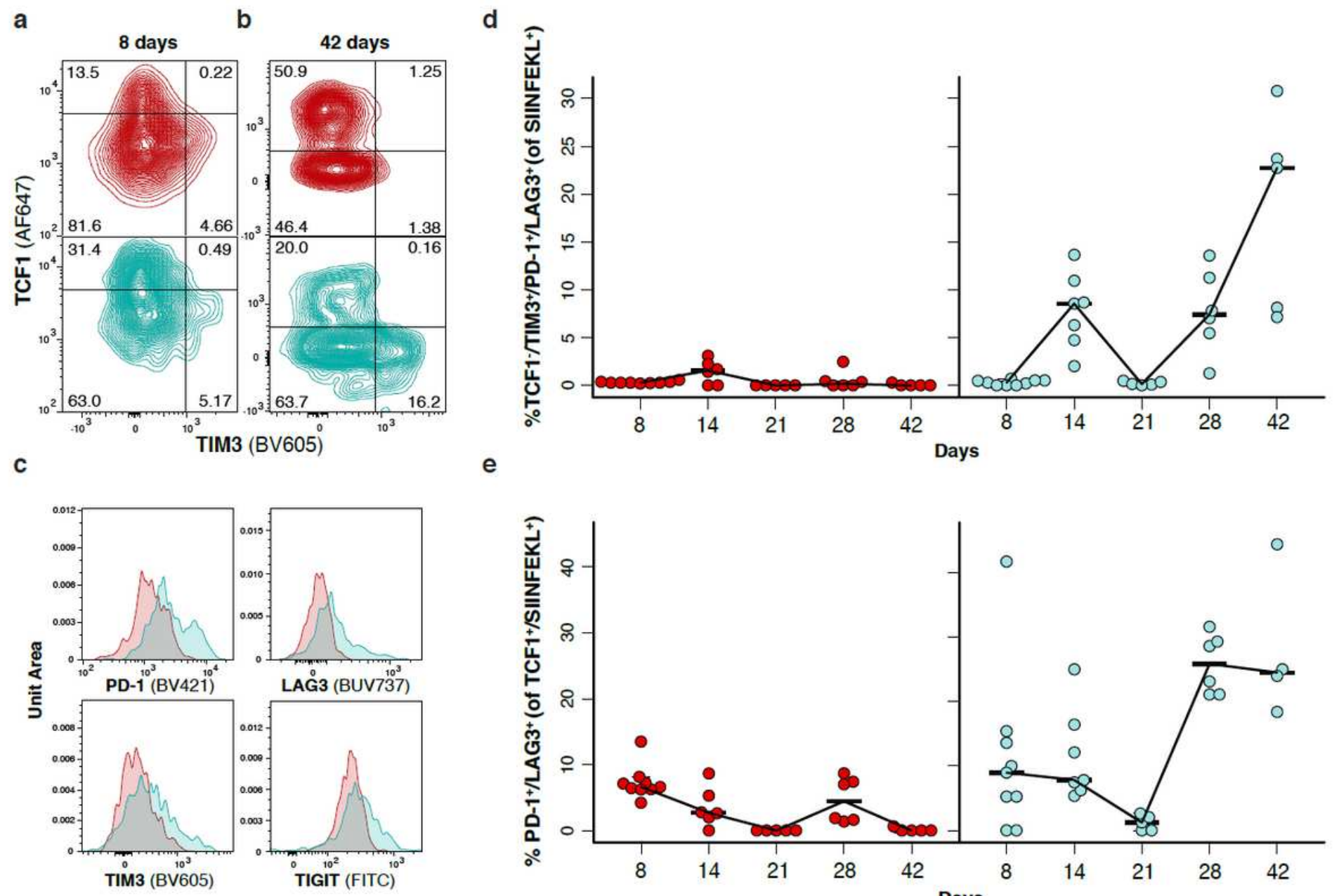

e

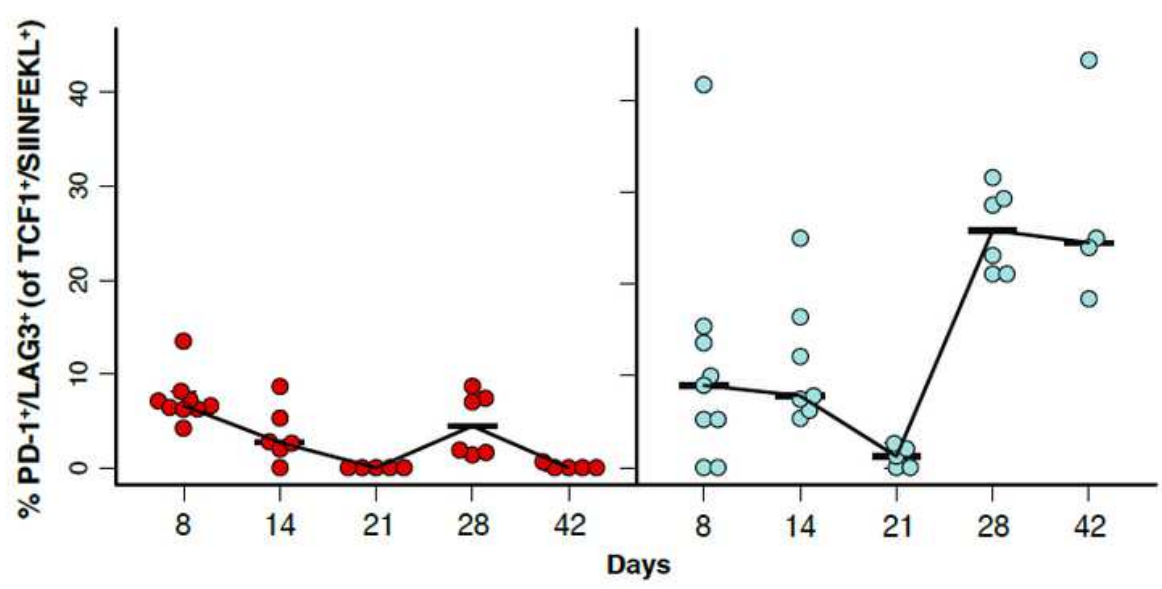

\section{Figure 3}

T cells in tumors with low neoantigen expression become progressively dysfunctional. (a-b) Antigenspecific T cell expression of TCF1 versus TIM3 in tumors at 8 (a) and 42 (b) days post-transplant. (c) Representative histograms of inhibitory receptor expression on antigen-specific $T$ cells from tumors at 42 days post-transplant. (d-e) Percent TCF1-/TIM3+/PD-1+/LAG3+ (d) and TCF1+/PD-1+/LAG3+ (e) antigenspecific $T$ cells isolated from tumors at $8,14,21,28$, and 42 days post-transplant. Red $=$ hiSIIN, blue $=$ IoSIIN. 
a
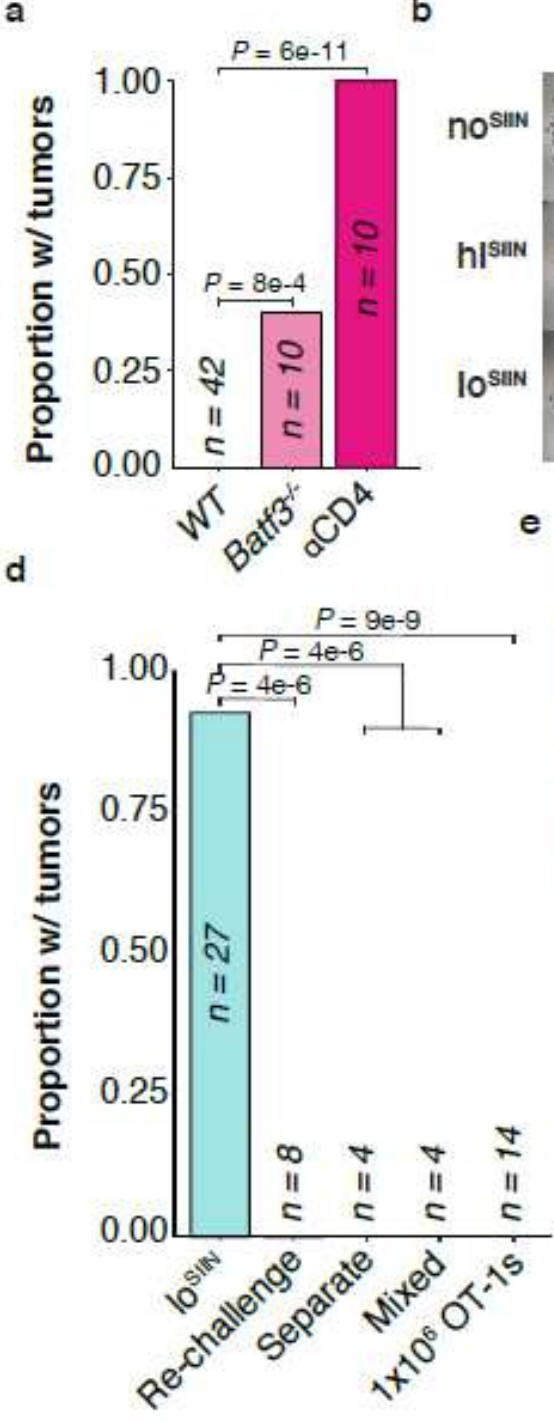

h

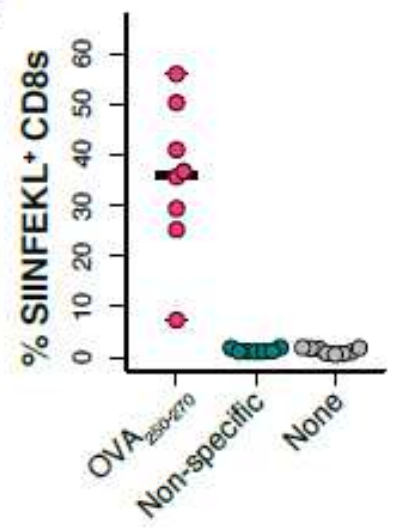

i

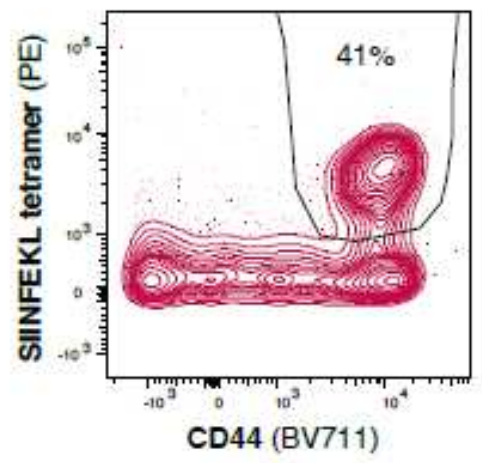

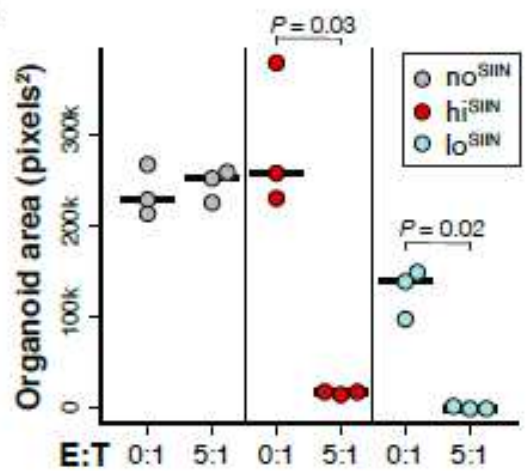

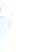
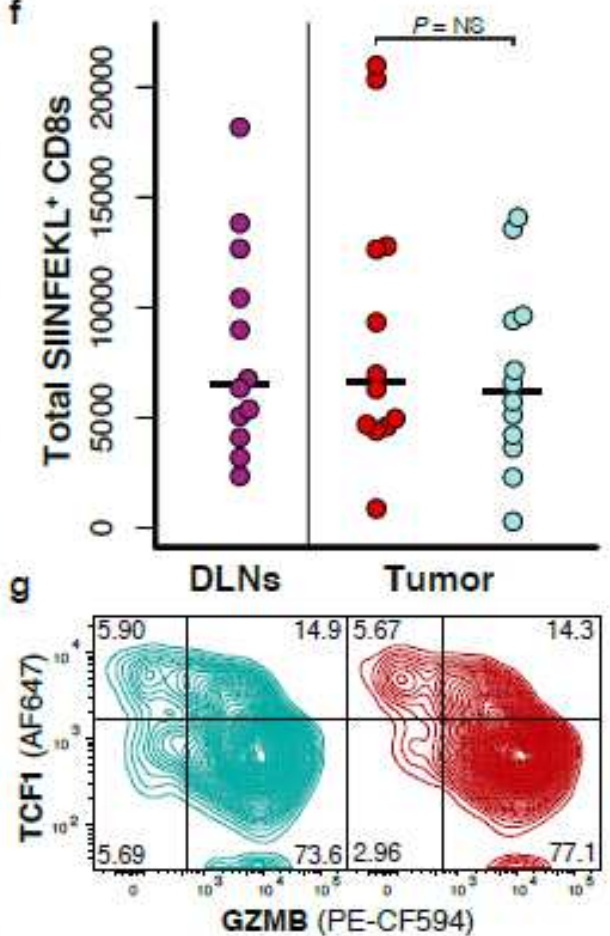

j

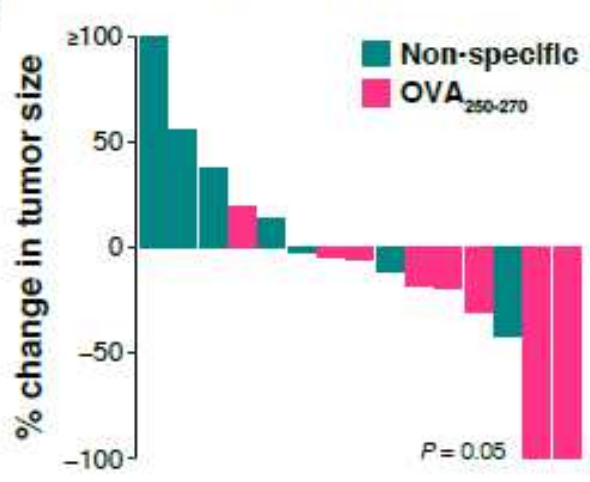

Figure 4

Low neoantigen expression results in poor $\mathrm{T}$ cell priming that can be rescued by therapeutic vaccination.

(a) Efficiency of tumor formation 6 weeks post-transplant of hiSIIN organoids into wild-type (WT) and Batf3 knockout mice, and 3 weeks post-transplant into WT mice with continuous antibody depletion of CD4+ T cells. (b) Representative images of co-cultures with noSIIN, hiSIIN, and IoSIIN organoids and in vitro-activated OT-1s at day 4. (c) Quantification of co-culture organoid confluence. E:T = effector to target 
ratio. (d) Efficiency of tumor formation 6 weeks post-transplant of loSIIN, loSIIN 4 weeks after transplant of hiSIIN (Re-challenge), loSIIN and hiSIIN at separate sites in the same animal (Separate), IoSIIN and hiSIIN at the same sites (Mixed), and loSIIN concurrent with retro-orbital injection of 1x106 in vitroactivated OT-1s. (e) Schematic of transplant of loSIIN (green) and hiSIIN (red) organoids at separate sites in the distal colon of the same animal (top), and stereoscopic brightfield (middle) and fluorescent (bottom) images of a co-transplanted colon 8 days post-transplant. (f-g) Flow cytometric analysis of antigen-specific T cells isolated from DLNs and tumors 8 days post-co-transplant of hiSIIN (red) and loSIIN (blue) organoids at separate sites in the same animals. (f) Total number of antigen-specific $T$ cells. (g) Representative expression of GZMB versus TCF1. (h) Percent of total peripheral blood CD8+ T cells that are antigen specific (CD44+/SIINFEKL tetramer+) following two weeks (two doses) of OVA250-270, non-specific, or no peptide-based vaccination in loSIIN tumor-bearing mice. (i) Representative flow plot of peripheral blood antigen-specific CD8+ T cells from OVA250-270 vaccinated mouse. (j) Change in loSIIN tumor size as measured by longitudinal colonoscopy following two weeks (two doses) of OVA250-270 or non-specific vaccination. Significance was assessed by Wilcoxon rank-sum test of percent change in tumor size.
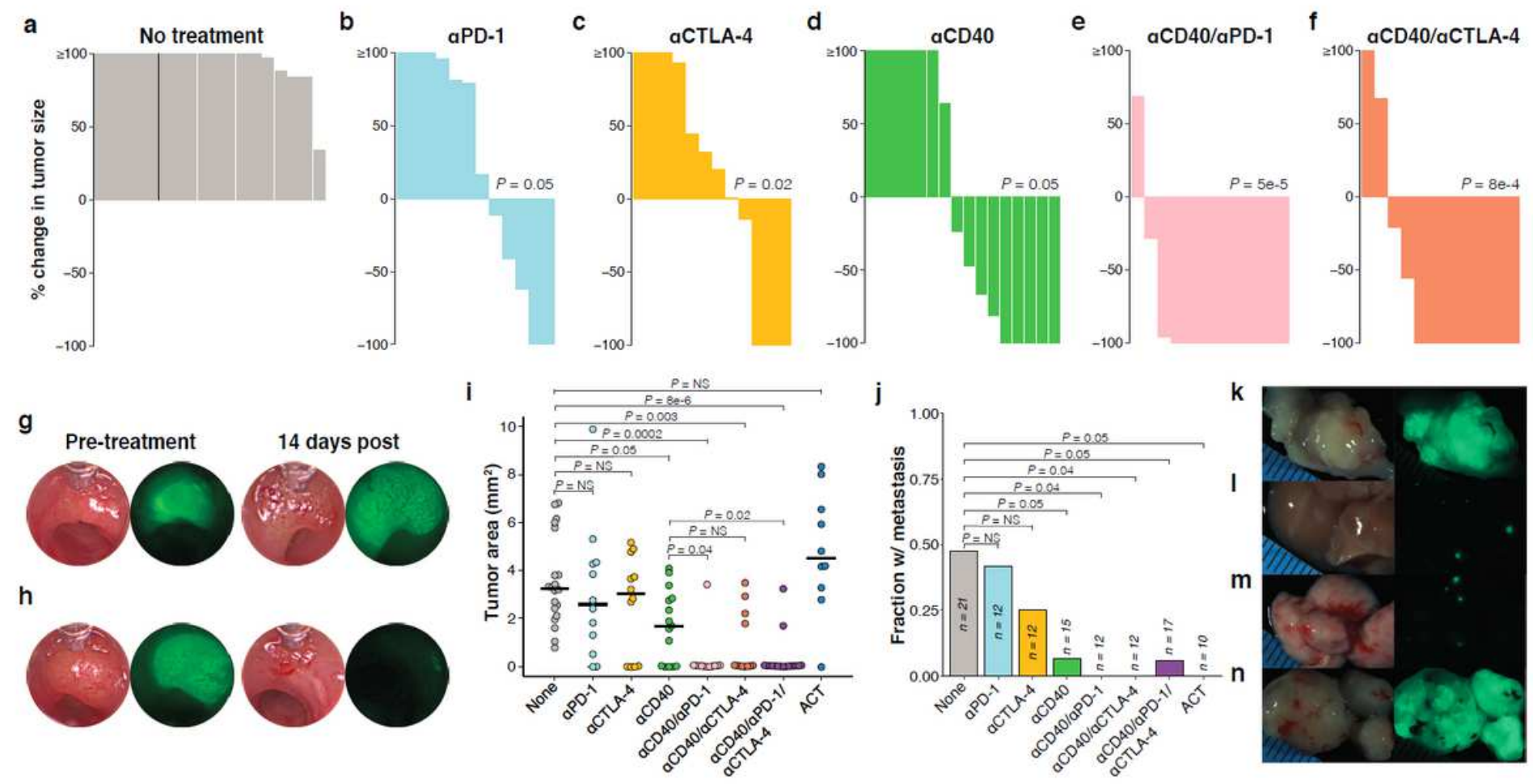

\section{Figure 5}

Agonistic CD40 rescues deficient priming and enhances checkpoint blockade in low neoantigenexpressing tumors. (a-f) Preclinical trial of mice bearing loSIIN tumors. Mice were recruited into treatment arms at 14 days post-transplant. Waterfall plots show change in tumor size after 14 days of treatment, as determined by colonoscopy. (g-h) Representative colonoscopy white light and fluorescent images of tumors pre- and post-treatment from mice receiving no treatment $(\mathrm{g})$ and aCD40/aPD-1/aCTLA-4 (h). (i) Primary tumor sizes at necropsy 28 days post-treatment initiation. ACT $=$ adoptive cell transfer of OT-1s. 
(j) Fraction of mice with any metastases (liver, lung, or omentum). (k-n) Representative stereoscopic white light and fluorescent images of primary colon tumor $(k)$, liver $(I)$, lung $(m)$, and omental $(n)$ metastases from an aPD-1-treated mouse 28 days post-treatment initiation.

\section{Supplementary Files}

This is a list of supplementary files associated with this preprint. Click to download.

- Flowcytometrysortingstrategyfigure.pdf 